\title{
Synthesis of Gentamicin Minor Components: Gentamicin B1 and Gentamicin X2
}

\author{
Parasuraman Rajasekaran ${ }^{\mathrm{a}, \mathrm{b}}$ and David Crich ${ }^{\mathrm{a}, \mathrm{b}, \mathrm{c}, *}$
}

a) Department of Pharmaceutical and Biomedical Sciences, University of Georgia, 250 West Green Street, Athens, GA 30602, USA

b) Complex Carbohydrate Research Center, University of Georgia, 315 Riverbend Road, Athens, GA 30602, USA

c) Department of Chemistry, University of Georgia, 140 Cedar Street, Athens, GA 30602, USA, 
Table of Contents

\begin{tabular}{|c|c|c|}
\hline & Expt & Spectra \\
\hline General experimental & S3 & \\
\hline $\begin{array}{l}\text { Methyl 2,3,4-tri- } O \text {-benzyl-7-deoxy- } \alpha \text {-L-glycero-D-gluco- } \\
\text { heptopyranoside (28). }\end{array}$ & S4 & S13-S14 \\
\hline $\begin{array}{l}\text { Methyl 2,3-di-O-acetyl-4,6-O-benzylidene-7-deoxy- } \alpha \text {-L-glycero-D- } \\
\text { gluco-heptopyranoside (S1). }\end{array}$ & S4-S5 & S15-S17 \\
\hline $\begin{array}{l}\text { Methyl 6-azido-2,3,4-tri- } O \text {-benzyl-6,7-dideoxy- } \alpha \text {-D-glycero-D- } \\
\text { gluco-heptopyranoside (29). }\end{array}$ & S5-S6 & S18-S19 \\
\hline $\begin{array}{l}p \text {-Tolyl 6-azido-2,3,4-tri- } O \text {-benzyl-6,7-dideoxy- } \alpha, \beta \text {-D-glycero-D- } \\
\text { gluco-heptothiopyranoside (30). }\end{array}$ & S6 & S20-S21 \\
\hline $\begin{array}{l}\text { 1,3,2',6'-Tetra(deamino)-5,2"-di-O-benzyl-1,3,2', } \text { ' }^{\prime} \text {-tetraazido-3"- } \\
N, 4^{\prime \prime}-O \text {-carbonyl-sisomicin (25). }\end{array}$ & S7 & S22-S23 \\
\hline $\begin{array}{l}\text { 5,2'-Di-O-benzyl-1,3-di(deamino)-1,3-diazido-3'- } N, 4^{\prime} \text {-O-carbonyl - } \\
\text { garamine (26). }\end{array}$ & S7-S8 & S24-S25 \\
\hline $\begin{array}{l}5,2^{\prime}, 3^{\prime}, 4^{\prime}, 2^{\prime \prime} \text {-Penta- } O \text {-benzyl-1,3,6'-triazido-1,3,6'-tri(deamino)-3"- } \\
N, 4^{\prime \prime}-O \text {-carbonyl-gentamicin B1 (31). }\end{array}$ & S8-S9 & S26-S27 \\
\hline Gentamicin B1 tetraaacetate salt (7). & S9 & S30-S31 \\
\hline $\begin{array}{l}5,3^{\prime}, 4^{\prime}, 6^{\prime}, 2^{\prime \prime} \text {-Penta-O-benzyl-1,3,2'-triazido-1,3,2'-tri(deamino)-3"- } \\
N, 4^{\prime \prime}-O \text {-carbonyl-gentamicin X2 (33). }\end{array}$ & S10 & S28-S29 \\
\hline Gentamicin X2 tetraaacetate salt (8). & S11 & S32-S33 \\
\hline References. & S12 & \\
\hline
\end{tabular}




\section{General Experimental}

All experiments were carried out under a dry argon atmosphere unless otherwise specified. Compounds that only appear in the Supporting Information have numbers prefaced with S.. Heating of reaction mixtures was carried out on an aluminum heating block of appropriate size. Chromatographic purifications were carried over silica gel (230-400 mesh). Thin layer chromatography was performed with precoated glass backed plates (w/UV 254). TLC were visualized by UV irradiation $(254 \mathrm{~nm})$ and by charring with sulfuric acid in ethanol (20:80, v/v) or with ceric ammonium molybdate solution [Ce( $\left(\mathrm{SO}_{4}\right)_{2}: 4 \mathrm{~g},\left(\mathrm{NH}_{4}\right)_{6} \mathrm{Mo}_{7} \mathrm{O}_{24}: 10 \mathrm{~g}, \mathrm{H}_{2} \mathrm{SO}_{4}: 40 \mathrm{~mL}$, $\mathrm{H}_{2} \mathrm{O}: 360 \mathrm{~mL}$ ]. Optical rotations were measured at $589 \mathrm{~nm}$ and $21^{\circ} \mathrm{C}$ on a digital polarimeter with a path length of $10 \mathrm{~cm}$. NMR spectra were recorded in $\mathrm{CDCl}_{3}$ or $\mathrm{D}_{2} \mathrm{O}$ using a 500, 600 or 900 $\mathrm{MHz}$ instrument, and assignments were made with the help of COSY, HMBC, and HSQC spectra. High-resolution (HRMS) mass spectra were recorded in the electrospray mode using a Orbitrap. Chemical shifts $(\delta)$ are recorded in ppm and multiplicities are abbreviated as follows: s (singlet), d (doublet), t (triplet), q (quartet), m (multiplet), and br (broad). 
Methyl 2,3,4-tri-O-benzyl-7-deoxy-a-L-glycero-D-gluco-heptopyranoside (28). To a stirred

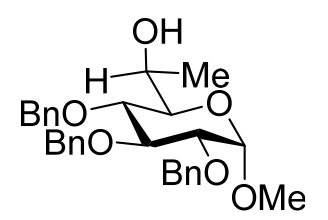
solution of $27^{1}(1.3 \mathrm{~g}, 2.79 \mathrm{mmol})$ in dry $\mathrm{CH}_{2} \mathrm{Cl}_{2}(20 \mathrm{~mL})$ at $0{ }^{\circ} \mathrm{C}$ was added Dess Martin periodinane $(1.42 \mathrm{~g}, 3.35 \mathrm{mmol})$. The resulting mixture was brought to room temperature and stirred for $2 \mathrm{~h}$ then quenched with $20 \%$ aqueous $\mathrm{Na}_{2} \mathrm{~S}_{2} \mathrm{O}_{3}$ and extracted with $\mathrm{CH}_{2} \mathrm{Cl}_{2}$. The organic layer was dried over $\mathrm{Na}_{2} \mathrm{SO}_{4}$ and evaporated to dryness, and the crude compound was used for the next step without further purification. The crude aldehyde was dissolved in THF $(20 \mathrm{~mL})$ and cooled to $-78^{\circ} \mathrm{C}$. The solution was treated with methylmagnesium chloride solution (3 $\mathrm{M}$ in THF, $2.79 \mathrm{~mL}, 8.37 \mathrm{mmol}$ ), and the resulting solution was stirred with gradual warming to room temperature. Saturated $\mathrm{NH}_{4} \mathrm{Cl}$ was added carefully, and the contents were extracted using EtOAc, dried $\left(\mathrm{Na}_{2} \mathrm{SO}_{4}\right)$, and concentrated using a rotary evaporator. The crude product was purified using silica gel column chromatography (eluent: 30 - $40 \%$ hexane/EtOAc) to give 28 (0.75 g, $56 \%$ overall for the two steps) in the form of a colorless oil; $[\alpha]_{\mathrm{D}}^{22}=+70.9\left(c 1.1, \mathrm{CHCl}_{3}\right) ;{ }^{1} \mathrm{H} \mathrm{NMR}\left(600 \mathrm{MHz}, \mathrm{CDCl}_{3}\right) \delta 7.39-7.25(\mathrm{~m}$, $15 \mathrm{H}), 5.00-4.91(\mathrm{~m}, 2 \mathrm{H}), 5.86-4.79(\mathrm{~m}, 2 \mathrm{H}), 4.70-4.65\left(\mathrm{~m}, 2 \mathrm{H}, \mathrm{PhCH}_{2}\right), 4.60(\mathrm{~d}, J=3.6 \mathrm{~Hz}$, 1H, H-1), 4.05 (q, $J=6.6 \mathrm{~Hz}, 1 \mathrm{H}, \mathrm{H}-6), 4.00$ (t, $J=9.3 \mathrm{~Hz}, 1 \mathrm{H}, \mathrm{H}-3), 3.63$ (dd, $J=10.0,9.0 \mathrm{~Hz}$, $1 \mathrm{H}, \mathrm{H}-4), 3.50$ (dd, $J=9.6,3.6 \mathrm{~Hz}, 1 \mathrm{H}, \mathrm{H}-2), 3.43$ (dd, $J=9.9,1.5 \mathrm{~Hz}, 1 \mathrm{H}, \mathrm{H}-5), 3.35$ (s, 3H, $\mathrm{OMe}), 1.25\left(\mathrm{~d}, J=6.6 \mathrm{~Hz}, 3 \mathrm{H}, \mathrm{CH}_{3}\right) .{ }^{13} \mathrm{C} \mathrm{NMR}\left(151 \mathrm{MHz}, \mathrm{CDCl}_{3}\right) \delta 138.9,138.4,138.3,128.6$, 128.3, 128.1, 128.0, 127.9, 127.7, 98.3, 82.3, 80.0, 77.8, 75.8, 75.2, 73.5, 72.9, 65.2, 55.1, 20.3. ESI-HRMS: m/z calcd. for $\mathrm{C}_{29} \mathrm{H}_{34} \mathrm{O}_{6} \mathrm{Na}[\mathrm{M}+\mathrm{Na}]^{+}$501.2253; found, 501.2237.
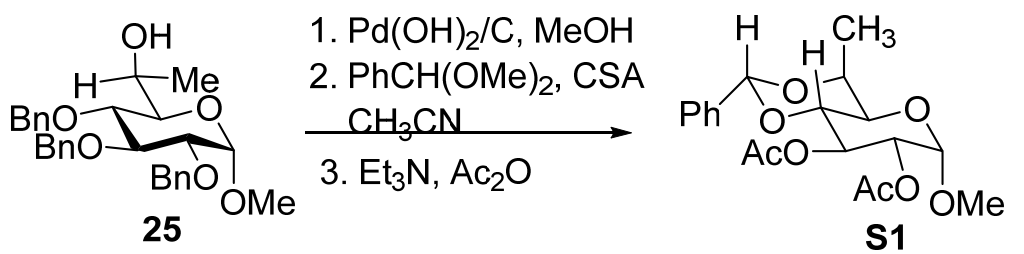

\section{Methyl 2,3-di-O-acetyl-4,6-O-benzylidene-7-deoxy- $\alpha$-L-glycero-D-gluco-heptopyranoside

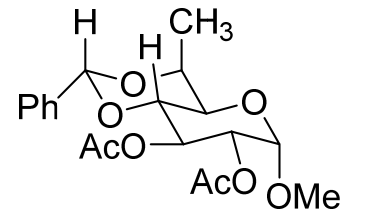 \\ (S1). ${ }^{2}$ A suspension of compound $28(60 \mathrm{mg}, 0.13 \mathrm{mmol})$ and $\mathrm{Pd}(\mathrm{OH})_{2} / \mathrm{C}$ $(10 \%, 15 \mathrm{mg})$ in methanol $(1.0 \mathrm{~mL})$ was subjected to hydrogenolysis under $45 \mathrm{psi}$ of $\mathrm{H}_{2}$ for $12 \mathrm{~h}$. After completion of the reaction, the catalyst was} filtered off and the filtrate was concentrated to dryness. To the crude mixture in $\mathrm{CH}_{3} \mathrm{CN}(0.8 \mathrm{~mL})$ benzaldehyde dimethyl acetal $(30 \mu \mathrm{L}, 0.19 \mathrm{mmol})$ was added followed by camphor-1-sulfonic acid (CSA, $3 \mathrm{mg}, 0.02 \mathrm{mmol}$ ). The reaction mixture was stirred at room temperature for $0.5 \mathrm{~h}$. After 
completion, the reaction was quenched with $\operatorname{Et}_{3} \mathrm{~N}(0.1 \mathrm{~mL})$, the solvent was evaporated to dryness and the residue was re-dissolved in mixture $\operatorname{Et}_{3} \mathrm{~N}: \mathrm{Ac}_{2} \mathrm{O}(1: 1,0.5 \mathrm{~mL})$ at $0{ }^{\circ} \mathrm{C}$ and stirred for $4 \mathrm{~h}$ at room temperature. After completion of the reaction, as indicated by TLC, the reaction mixture was diluted with $\mathrm{CH}_{2} \mathrm{Cl}_{2}$, washed (sat. aq. $\left.\mathrm{NaHCO}_{3}\right)$, dried $\left(\mathrm{Na}_{2} \mathrm{SO}_{4}\right)$, and concentrated. Purification of the crude compound by column chromatography (eluent: 20 - 30 \% hexane/EtOAc) afforded S1 (35 mg, $72 \%$ ) as a gum. $[\alpha]_{\mathrm{D}}{ }^{22}=+71.9\left(c 0.53, \mathrm{CHCl}_{3}\right) ;{ }^{1} \mathrm{H} \mathrm{NMR}\left(500 \mathrm{MHz}, \mathrm{CDCl}_{3}\right) \delta 7.48-$ 7.39 (m, 2H), $7.38-7.30$ (m, 3H), 5.79 (s, 1H, PhCH), 5.57 (t, $J=9.8 \mathrm{~Hz}, 1 \mathrm{H}, \mathrm{H}-3), 4.92$ (d, $J=$ $3.7 \mathrm{~Hz}, 1 \mathrm{H}, \mathrm{H}-1), 4.87$ (dd, $J=10.0,3.7 \mathrm{~Hz}, 1 \mathrm{H}, \mathrm{H}-2), 4.49$ (p, $J=6.7 \mathrm{~Hz}, 1 \mathrm{H}, \mathrm{H}-6), 4.15$ (dd, $J$ $=10.3,5.9 \mathrm{~Hz}, 1 \mathrm{H}, \mathrm{H}-5), 3.91$ (t, $J=9.9 \mathrm{~Hz}, 1 \mathrm{H}, \mathrm{H}-4), 3.41$ (s, 3H, OMe), 2.09 (s, 3H, $\left.\mathrm{CH}_{3} \mathrm{CO}\right)$, $2.05\left(\mathrm{~s}, 3 \mathrm{H}, \mathrm{CH}_{3} \mathrm{CO}\right), 1.47\left(\mathrm{~d}, J=6.9 \mathrm{~Hz}, 3 \mathrm{H}, \mathrm{CH}_{3}\right) ;{ }^{13} \mathrm{C} \mathrm{NMR}\left(125 \mathrm{MHz}, \mathrm{CDCl}_{3}\right) \delta$ 170.5, 169.7, $137.4,128.9,128.2,126.2,97.5,94.0,72.7,71.5,70.5$, 69.6, 64.4, 55.3, 20.8, 20.8, 11.3; ESIHRMS: $\mathrm{m} / \mathrm{z}$ calcd. for $\mathrm{C}_{19} \mathrm{H}_{24} \mathrm{O} 8 \mathrm{Na}[\mathrm{M}+\mathrm{Na}]^{+} 403.1363$; found, 403.1360 .

Methyl 6-azido-2,3,4-tri-O-benzyl-6,7-dideoxy-a-D-glycero-D-gluco-heptopyranoside (29).

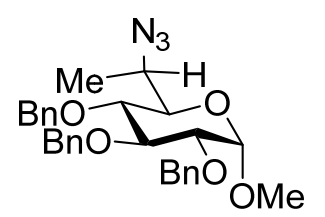

Alcohol $28(0.60 \mathrm{~g}, 1.26 \mathrm{mmol})$ and $\mathrm{PPh}_{3}(0.39 \mathrm{~g} 1.51 \mathrm{mmol})$ were dissolved in THF $(10 \mathrm{~mL})$, diisopropylethylamine $(0.21 \mathrm{~mL} 20.8 \mathrm{mmol})$ was added and the mixture cooled to $10^{\circ} \mathrm{C}$. Diisopropyl azodicarboxylate (DIAD, $0.3 \mathrm{~mL}$,

$1.51 \mathrm{mmol}$ ) was then added and the reaction mixture stirred for $10 \mathrm{~min}$.

Diphenylphosphoryl azide (DPPA, $0.39 \mathrm{~mL} 1.51 \mathrm{mmol}$ ) was then added at same temperature and the reaction mixture was warmed to room temperature and stirred for $2 \mathrm{~h}$. It was quenched with satd. aq. $\mathrm{NH}_{4} \mathrm{Cl}$ and extracted using EtOAc, dried over $\mathrm{Na}_{2} \mathrm{SO}_{4}$ and concentrated under reduced pressure. The crude product was purified using silica gel column chromatography (eluent: $10-$ $15 \%$ hexane/EtOAc) to give $29(0.52 \mathrm{~g}, 83 \%)$, in the form of a colorless oil. $[\alpha]_{\mathrm{D}}^{22}=+16.3(c 0.60$, $\left.\mathrm{CH}_{2} \mathrm{Cl}_{2}\right) ;{ }^{1} \mathrm{H} \mathrm{NMR}\left(600 \mathrm{MHz}, \mathrm{CDCl}_{3}\right) \delta 7.47-7.06(\mathrm{~m}, 15 \mathrm{H}), 5.04(\mathrm{~d}, J=10.8 \mathrm{~Hz}, 1 \mathrm{H}), 4.92(\mathrm{~d}$, $J=11.2 \mathrm{~Hz}, 1 \mathrm{H}), 4.87-4.78(\mathrm{~m}, 2 \mathrm{H}), 4.70(\mathrm{~d}, J=12.1 \mathrm{~Hz}, 1 \mathrm{H}), 4.65$ (d, $J=3.6 \mathrm{~Hz}, 1 \mathrm{H}, \mathrm{H}-1)$, $4.61(\mathrm{~d}, J=11.2 \mathrm{~Hz}, 1 \mathrm{H}), 4.02$ (dd, $J=9.6,8.7 \mathrm{~Hz}, 1 \mathrm{H}, \mathrm{H}-3), 3.87$ (dd, $J=10.2,2.1 \mathrm{~Hz}, 1 \mathrm{H}, \mathrm{H}-$ 5), 3.58 (qd, $J=6.9,2.1 \mathrm{~Hz}, 1 \mathrm{H}, \mathrm{H}-6$ ), 3.52 (dd, $J=9.6,3.7 \mathrm{~Hz}, 1 \mathrm{H}, \mathrm{H}-2), 3.45$ (s, 3H), 3.34 (dd, $J=10.2,8.7 \mathrm{~Hz}, 1 \mathrm{H}, \mathrm{H}-4), 1.12(\mathrm{~d}, J=6.9 \mathrm{~Hz}, 3 \mathrm{H}) ;{ }^{13} \mathrm{C} \mathrm{NMR}\left(151 \mathrm{MHz}, \mathrm{CDCl}_{3}\right) \delta 138.6,138.0$, $137.8,128.5,128.5,128.5,128.1,128.1,128.1,128.0,128.0,128.0,127.7,97.8,82.3,80.1,77.7$, 
75.8, 74.6, 73.3, 72.0, 55.7, 55.3, 11.9; ESI-HRMS: $\mathrm{m} / \mathrm{z}$ calcd. for $\mathrm{C}_{29} \mathrm{H}_{33} \mathrm{O}_{5} \mathrm{~N}_{3} \mathrm{Na}[\mathrm{M}+\mathrm{Na}]^{+}$ 526.2312; found, 526.2310.

\section{$p$-Tolyl 6-azido-2,3,4-tri- $O$-benzyl-6,7-dideoxy- $\alpha, \beta$-D-glycero-D-gluco-heptothiopyranoside}

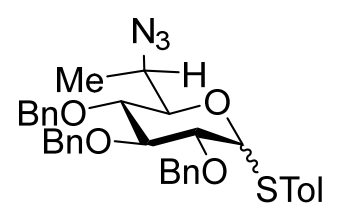

(30). To a solution of $29(200 \mathrm{mg}, 0.40 \mathrm{mmol})$ and acetic anhydride $(200 \mu \mathrm{L})$ in acetic acid $(3 \mathrm{~mL}), \mathrm{H}_{2} \mathrm{SO}_{4}(50 \mu \mathrm{L})$ was added at $0{ }^{\circ} \mathrm{C}$. The reaction mixture

was stirred for $1 \mathrm{~h}$ at r.t., and then quenched with water, diluted with $\mathrm{CH}_{2} \mathrm{Cl}_{2}$,

washed (sat. aq. $\mathrm{NaCO}_{3}$, and water), dried $\left(\mathrm{Na}_{2} \mathrm{SO}_{4}\right)$, and concentrated. A quick filtration of the crude reaction mixture through a pad of silica gel (eluent: 20 - 30\% hexane/EtOAc) gave a mixture of the anomeric acetates. To this mixture in $\mathrm{CH}_{2} \mathrm{Cl}_{2}(3 \mathrm{~mL})$, activated $4 \AA$ powdered molecular sieves, and TolSH (99 mg, $0.8 \mathrm{mmol}$ ) were added, followed by slow addition of BF 3 . OEt ( $60 \mu \mathrm{L}$, $0.48 \mathrm{mmol}$ ) at $0{ }^{\circ} \mathrm{C}$. The reaction mixture was stirred for $1 \mathrm{~h}$ at $0{ }^{\circ} \mathrm{C}$, and an additional $12 \mathrm{~h}$ at room temperature, then was diluted with $\mathrm{CH}_{2} \mathrm{Cl}_{2}$, washed (sat. aq. $\mathrm{NaHCO}_{3}$ ), dried $\left(\mathrm{Na}_{2} \mathrm{SO}_{4}\right.$ ), and concentrated. The crude product was purified using silica gel column chromatography (eluent: 5 $-10 \%$ hexane/EtOAc) to give an anomeric mixture of $30(161 \mathrm{mg}, 68 \%, \alpha / \beta=3 / 1)$ as a colorless oil. ${ }^{1} \mathrm{H}$ NMR (600 MHz, $\left.\mathrm{CDCl}_{3}\right) \delta 7.53-7.10$ (m, 19H, both anomers), 5.58 (d, $J=5.4 \mathrm{~Hz}, 1 \mathrm{H}, \alpha$ anomer), $5.07-4.63(\mathrm{~m}, 6 \mathrm{H}$, both anomers ), $4.61(\mathrm{~d}, J=9.8 \mathrm{~Hz}, 1 \mathrm{H}, \beta$ isomer $), 4.43-4.38(\mathrm{~m}$, 1H), 3.93 (dd, $J=9.6,8.6 \mathrm{~Hz}, 1 \mathrm{H}, \alpha$ anomer), 3.84 (dd, $J=9.6,5.4 \mathrm{~Hz}, 1 \mathrm{H}, \alpha$ anomer), 3.73 (t, $J$ $=8.8 \mathrm{~Hz}, \beta$ isomer $), 3.69-3.52(\mathrm{~m}, 1 \mathrm{H}, \alpha$ anomer, $2 \mathrm{H}, \beta$ isomer $), 3.50-3.43(\mathrm{~m}, 1 \mathrm{H}, 2 \mathrm{H}, \beta$ isomer), 3.41 (dd, $J=10.2,8.6 \mathrm{~Hz}, 1 \mathrm{H}, \alpha$ anomer), 2.37 (s,3H, $\beta$ isomer), 2.36 (s, $3 \mathrm{H}, \alpha$ anomer), $1.20(\mathrm{~d}, J=6.9 \mathrm{~Hz}, 1 \mathrm{H}, \beta$ isomer $), 1.13(\mathrm{~d}, J=6.9 \mathrm{~Hz}, 3 \mathrm{H}, \alpha$ anomer $),{ }^{13} \mathrm{C} \mathrm{NMR}\left(151 \mathrm{MHz}, \mathrm{CDCl}_{3}\right)$ $\delta 149.9,138.5,138.2,138.1,138.0,137.8,137.6,133.2,132.6,130.1,130.1,129.8,129.8,129.7$, $128.6,128.5,128.5,128.5,128.5,128.2,128.1,128.1,128.1,128.0,128.0,127.9,127.8,127.8$, 127.7, 126.1, 120.3, 120.2, 87.9, 87.5, 87.1, 82.6, 80.9, 80.6, 79.9, 77.6, 77.4, 75.8, 75.8, 75.4, 74.7, 74.6, 73.2, 72.3, 56.2, 56.0, 21.1, 12.4, 12.4; .ESI-HRMS: m/z calcd. for $\mathrm{C}_{35} \mathrm{H}_{37} \mathrm{O}_{4} \mathrm{~N}_{3} \mathrm{Na}$ $[\mathrm{M}+\mathrm{Na}]^{+}$618.2402; found, 618.2401. 


\section{$1,3,2^{\prime}, 6^{\prime}$-Tetra(deamino)-5,2"'-di- $O$-benzyl-1,3,2',6'-tetraazido-3"'- $N, 4^{\prime \prime}-O$-carbonyl-}

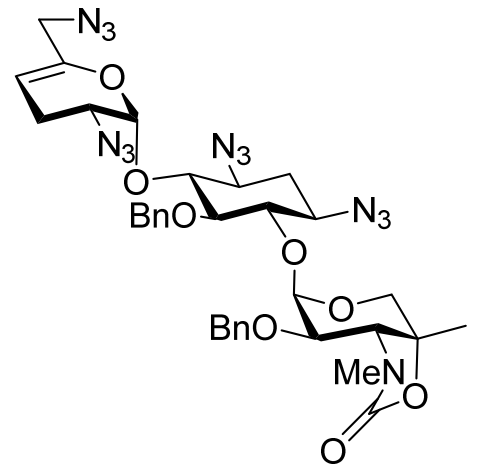

sisomicin (25). A stirred solution of 1,3,2',6'-tetra-deamino-3"'-Nbenzyloxycarbonyl-1,3,2',6'-tetraazido-sisomicin $\mathbf{2 4}^{3}$ (660 mg, 0.88 mmol) in DMF $(8 \mathrm{~mL})$ cooled to $0{ }^{\circ} \mathrm{C}$ was treated with $\mathrm{NaH}(105$ $\mathrm{mg}, 60 \%$ in paraffin oil, $2.63 \mathrm{mmol}$ ) and stirred at same temperature for $20 \mathrm{~min}$. After disappearance of the starting materials based on TLC, benzyl bromide $(0.45 \mathrm{~mL}, 3.50 \mathrm{mmol})$ was added dropwise. The resulting reaction mixture was allowed to stir at room temperature for $2 \mathrm{~h}$ before the reaction was quenched by addition of a saturated aqueous solution of $\mathrm{NH}_{4} \mathrm{Cl}$. The solvents were evaporated and the crude product was purified using silica gel column chromatography (eluent: $10-12 \%$ hexane/EtOAc) to give compound $25(635 \mathrm{mg}, 87 \%)$ as a yellow oil. $[\alpha] \mathrm{D}^{21}=+68.0\left(c 0.25, \mathrm{CHCl}_{3}\right) ;{ }^{1} \mathrm{H} \mathrm{NMR}\left(600 \mathrm{MHz}, \mathrm{CDCl}_{3}\right) \delta 7.44-7.23(\mathrm{~m}, 10 \mathrm{H})$, 5.67 (d, $\left.J=2.5 \mathrm{~Hz}, 1 \mathrm{H}, \mathrm{H}-1^{\prime}\right), 5.16$ (d, $\left.J=3.4 \mathrm{~Hz}, 1 \mathrm{H}, \mathrm{H}-1^{\prime \prime}\right), 5.11$ (d, $J=10.8 \mathrm{~Hz}, 1 \mathrm{H}, \mathrm{PhCH}_{2}$ ), $4.93\left(\mathrm{dd}, J=5.4,2.4 \mathrm{~Hz}, 1 \mathrm{H}, \mathrm{H} 4\right.$ '), 4.88 (d, $\left.J=11.5 \mathrm{~Hz}, 1 \mathrm{H}, \mathrm{PhCH}_{2}\right), 4.74-4.64$ (m, 2H, $\mathrm{PhCH}_{2}$ ), 3.97 (t, J=3.5 Hz, 1H, H-2"), 3.82 - 3.63 (m, 5H, CH2, H-5a", H-4, H-5, CH2, H-6'a, H-6'b), 3.50 (t, $J=9.4$ Hz, 1H, H-6), 3.48 - 3.42 (m, 2H.H-1, H-3"), 3.40 - 3.34 (m, 2H, H-3, CH2, H-5b"), 3.27 (ddd, $J=10.7,6.1,2.5 \mathrm{~Hz}, 1 \mathrm{H}, \mathrm{H}-2), 2.79$ (s, 3H, NCH 3 ), 2.50 - 2.39 (m, 1H, H-3a"), 2.28 2.19 (m, 2H, H-3b", H-2a"), 1.41 (q, $J=12.8 \mathrm{~Hz}, 1 \mathrm{H}, \mathrm{H}-2 \mathrm{~b} "), 1.22$ (s, 3H, CH 3$) ;{ }^{13} \mathrm{C}$ NMR (151 $\left.\mathrm{MHz} \mathrm{CDCl}_{3}\right) \delta 157.4,145.7,138.1,137.4,128.6,128.3,128.2,128.2,128.0,127.5,126.9,126.9$, $97.9,96.6,96.3,82.8,80.7,78.4,77.0,75.1,75.0,72.3,67.0,63.2,61.2,60.3,54.6,52.2,32.5$, 29.9, 23.3, 21.2; m/z calcd. for $\mathrm{C}_{34} \mathrm{H}_{39} \mathrm{O}_{8} \mathrm{~N}_{13}[\mathrm{M}+\mathrm{Na}]^{+}$780.2942; found, 780.2939.

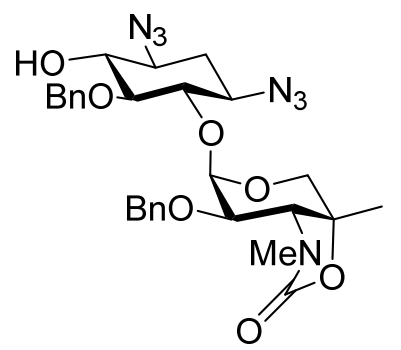

5,2'-Di-O-benzyl-1,3-di(deamino)-1,3-diazido-3'- $N, 4^{\prime}$-O $O$-carbonylgaramine (26). Compound 25 (600 $\mathrm{mg}, 0.79 \mathrm{mmol})$ was dissolved in tetrahydrofuran $(15 \mathrm{~mL})$, and the $\mathrm{pH}$ of the solution adjusted to 1 by careful addition of concentrated sulfuric acid in tetrahydrofuran $(2 \%$ solution of $\mathrm{H}_{2} \mathrm{SO}_{4}$ in THF, $1 \mathrm{~mL}$ was added). The solution was stirred at $40{ }^{\circ} \mathrm{C}$ for $48 \mathrm{~h}$, then was diluted with EtOAc, neutralized with aq. $\mathrm{NaHCO}_{3}$, extracted using EtOAc, dried over $\mathrm{Na}_{2} \mathrm{SO}_{4}$ and concentrated under reduced pressure. The crude product was purified using silica gel column chromatography (eluent: 30 - 40\% 
hexane/EtOAc) to give 26 (390 mg, 85\%), in the form of a colorless oil. $[\alpha]_{\mathrm{D}}^{21}=+19.2(c 0.25$, $\left.\mathrm{CHCl}_{3}\right) ;{ }^{1} \mathrm{H} \mathrm{NMR}\left(600 \mathrm{MHz}, \mathrm{CDCl}_{3}\right) \delta 7.41-7.23$ (m, 10H), 5.20 (d, $\left.J=3.5 \mathrm{~Hz}, 1 \mathrm{H}, \mathrm{H}-1 "\right), 5.00$ (d, $\left.J=11.4 \mathrm{~Hz}, 1 \mathrm{H}, \mathrm{PhCH}_{2}\right), 4.91\left(\mathrm{~d}, J=11.6 \mathrm{~Hz}, 1 \mathrm{H}, \mathrm{PhCH}_{2}\right), 4.66$ (dd, $J=11.5,3.0 \mathrm{~Hz}, 2 \mathrm{H}$, $\mathrm{PhCH}_{2}$ ), 3.98 (t, $\left.J=3.7 \mathrm{~Hz}, 1 \mathrm{H}, \mathrm{H}-2 "\right), 3.95$ (d, $\left.J=12.5 \mathrm{~Hz}, 1 \mathrm{H}, \mathrm{CH}_{2}, \mathrm{H}-5 \mathrm{a} "\right), 3.68$ (t, $J=9.6 \mathrm{~Hz}$, 1H, H-6), 3.54 - 3.42 (m, 4H, CH $2, \mathrm{H}-5 \mathrm{~b} ", \mathrm{H}-3$ ", H-4, H-1), 3.38 (ddd, $J=12.4,9.7,4.5$ Hz, 1H, H-3), 3.25 (t, $J=9.3 \mathrm{~Hz}, 1 \mathrm{H}, \mathrm{H}-5), 2.81$ (s, 3H, NCH3), 2.64 (d, $J=2.4 \mathrm{~Hz}, 1 \mathrm{H}, \mathrm{OH}), 2.20$ (dt, $J$ $=13.3,4.5 \mathrm{~Hz}, 1 \mathrm{H}, \mathrm{H}-2 \mathrm{a}), 1.39$ (q, $J=12.7 \mathrm{~Hz}, 1 \mathrm{H}, \mathrm{H}-2 \mathrm{~b}), 1.28$ (s, 3H, CH3). ${ }^{13} \mathrm{C}$ NMR $(151$ $\left.\mathrm{MHz}, \mathrm{CDCl}_{3}\right) \delta 157.4,138.1,137.5,128.6,128.6,128.2,127.9,127.8,127.5,96.1,81.9,80.3$, 77.3, 76.0, 75.3, 74.8, 74.8, 72.7, 66.7, 63.0, 61.3, 59.6, 32.0, 30.0, 23.3. ESI-HRMS: m/z calcd. for $\mathrm{C}_{28} \mathrm{H}_{34} \mathrm{O}_{7} \mathrm{~N}_{7}[\mathrm{M}+\mathrm{H}]^{+} 580.2520$; found, 580.2510 .

\section{$5,2^{\prime}, 3^{\prime}, 4^{\prime}, 2^{\prime \prime}$-Penta-O-benzyl-1,3,6'-triazido-1,3,6'-tri(deamino)-3"'-N,4'"-O-carbonyl-}

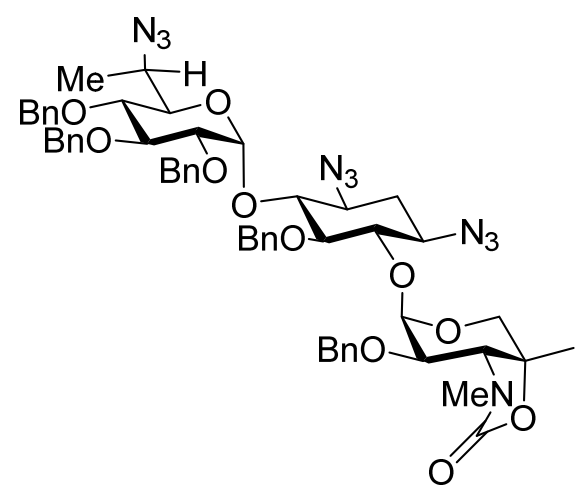

gentamicin B1 (31). A mixture of acceptor $26(57 \mathrm{mg}$; 0.10 $\mathrm{mmol}$ ), donor 30 (90 $\mathrm{mg}, 0.15 \mathrm{mmol}$ ), and freshly activated 4 $\AA$ acid-washed powdered molecular sieves in dichloromethane $(2.0 \mathrm{~mL})$ was stirred for $1 \mathrm{~h}$ at room temperature, then cooled to $-30{ }^{\circ} \mathrm{C}$, and treated with $\mathrm{N}$ iodosuccinimide ( $41 \mathrm{mg} ; 0.18 \mathrm{mmol}$ ) and $\mathrm{AgOTf}(8 \mathrm{mg} ; 0.03$ $\mathrm{mmol}$ ). The reaction mixture was stirred at $-30{ }^{\circ} \mathrm{C}$ for $1 \mathrm{~h}$ and then gradually warmed to room temperature and stirred for $6 \mathrm{~h}$ before it was quenched with triethylamine $(0.2 \mathrm{~mL})$. The mixture was diluted with dichloromethane, filtered through Celite ${ }^{\circledR}$, washed with $20 \%$ aqueous $\mathrm{Na}_{2} \mathrm{~S}_{2} \mathrm{O}_{3}$, dried over $\mathrm{Na}_{2} \mathrm{SO}_{4}$, and concentrated under reduced pressure. The crude product was purified using silica gel column chromatography (eluent: $25-35 \%$ hexane/EtOAc) to give 31 (75 mg, 76\%), in the form of a colorless oil. $[\alpha]_{\mathrm{D}}{ }^{21}=+17.3(c 0.53$, $\left.\mathrm{CH}_{2} \mathrm{Cl}_{2}\right) ;{ }^{1} \mathrm{H}$ NMR $\left(600 \mathrm{MHz}, \mathrm{CDCl}_{3}\right) \delta 7.40-7.18(\mathrm{~m}, 20 \mathrm{H}), 7.18-7.11(\mathrm{~m}, 2 \mathrm{H}), 7.04-6.96$ (m, 3H), 5.65 (d, J=3.8 Hz, 1H, H-1'), 5.18 (d, J=3.6 Hz, 1H, H-1"), $4.94-4.84$ (m, 4H, $\mathrm{PhCH}_{2}$ ), 4.78 (dd, $\left.J=11.2,1.9 \mathrm{~Hz}, 2 \mathrm{H}, \mathrm{PhCH}_{2}\right), 4.60$ (d, $\left.J=11.3 \mathrm{~Hz}, 2 \mathrm{H}, \mathrm{PhCH}_{2}\right), 4.50-4.37$ (m, 2H, $\mathrm{PhCH}_{2}$ ), 4.36 (dd, $\left.J=10.2,1.8 \mathrm{~Hz}, 1 \mathrm{H}, \mathrm{H}_{-} 5^{\prime}\right), 4.04$ (dd, $\left.J=9.9,8.8 \mathrm{~Hz}, 1 \mathrm{H}, \mathrm{H}-3^{\prime}\right), 3.92$ (t, $J=3.8$ Hz, 1H, H-2"), 3.77 (td, $J=9.6,2.5$ Hz, 2H, H-6, H-4), 3.64 - 3.52 (m, 3H, H-6', H-5, H-1), 3.52 - 3.44 (m, 2H, H-3, $\mathrm{CH}_{2}, \mathrm{H}-5 \mathrm{a}$ )), 3.41 (d, $\left.J=3.9 \mathrm{~Hz}, 1 \mathrm{H}, \mathrm{H}-3 "\right), 3.37$ (dd, $J=9.9,3.8 \mathrm{~Hz}, 1 \mathrm{H}, \mathrm{H}-$ 
2'), 3.33 (dd, $J=10.2,8.8 \mathrm{~Hz}, 1 \mathrm{H}, \mathrm{H}-4$ '), 3.09 (d, $J=12.8 \mathrm{~Hz}, 1 \mathrm{H}, \mathrm{CH}_{2}, \mathrm{H}-5 \mathrm{~b}$ )), 2.79 (s, 3H, $\mathrm{NCH}_{3}$ ), 2.35 (dt, $\left.J=13.2,4.4 \mathrm{~Hz}, 1 \mathrm{H}, \mathrm{H}-2 \mathrm{a}\right), 1.59$ (q, $\left.J=12.8 \mathrm{~Hz}, 1 \mathrm{H}, \mathrm{H}-2 \mathrm{~b}\right), 1.12$ (d, $J=7.0 \mathrm{~Hz}$, $3 \mathrm{H}), 1.01(\mathrm{~s}, 3 \mathrm{H}) .1 .12\left(\mathrm{~d}, J=6.9 \mathrm{~Hz}, 3 \mathrm{H}, \mathrm{CH}_{3}\right), 1.00\left(\mathrm{~s}, 3 \mathrm{H}, \mathrm{CH}_{3}\right) ;{ }^{13} \mathrm{C} \mathrm{NMR}\left(151 \mathrm{MHz}, \mathrm{CDCl}_{3}\right)$ $\delta 157.4,138.4,138.4,137.9,137.7,137.5,128.6,128.5,128.4,128.4,128.3,128.2,128.2,128.1$, $128.1,128.0,127.9,127.9,127.9,127.8,127.8,127.7,127.6,127.5,127.4,126.9,125.6,96.6$, 95.6, 82.8, 82.0, 79.7, 79.2, 78.0, 75.5, 74.8, 74.4, 73.7, 73.4, 72.9, 72.6, 66.2, 62.8, 61.1, 59.5, 56.8, 32.1, 30.0, 22.9, 12.9; ESI-HRMS: m/z calcd. for $\mathrm{C}_{56} \mathrm{H}_{62} \mathrm{O}_{11} \mathrm{~N}_{10} \mathrm{Na}[\mathrm{M}+\mathrm{Na}]^{+}$1073.4497; found, 1073.4480 .

Gentamicin B1 tetraaacetate salt (7). A suspension of compound 31 (23 mg, $21.9 \mu \mathrm{mol})$ and

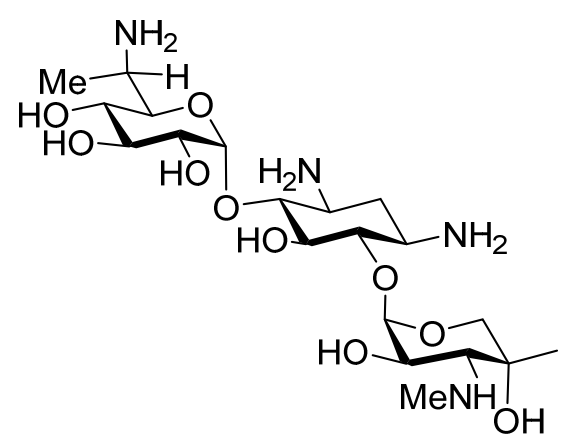
$\mathrm{Pd}(\mathrm{OH})_{2} / \mathrm{C}(10 \%, 50 \mathrm{mg})$ in 1,4-dioxane: $\mathrm{H}_{2} \mathrm{O}(1: 1,0.8 \mathrm{~mL})$ was subjected to hydrogenolysis under 40 psi of $\mathrm{H}_{2}$ for $36 \mathrm{~h}$. After completion of the reaction, the catalyst was filtered off and the filtrate was concentrated to dryness. The crude residue was re-dissolved in saturated aqueous $\mathrm{Ba}(\mathrm{OH})_{2}(0.5 \mathrm{~mL})$ and heated to $60{ }^{\circ} \mathrm{C}$ for $12 \mathrm{~h}$. After complete hydrolysis of the oxazolidinone, as indicated by mass spectrometry, the reaction mixture was neutralized to $\mathrm{pH} 7$ by addition of dry ice and the precipitated solid was filtered off. The filter cake was washed with water and the filtrate was concentrated to dryness. The crude product was then taken up in 10\% aqueous acetic acid and loaded on a prepacked Sephadex C-25 column and eluted with $0.1 \%$ to $1.5 \%$ ammonium hydroxide in deionized water. The productcontaining fractions were combined, concentrated, acidified with glacial acetic acid, and lyophilized to give the pentaacetate salt of gentamicin B1 (7) as a white foam (6.1 mg, $56 \%$ ). $[\alpha] \mathrm{D}^{21}=+30\left(c 0.1, \mathrm{H}_{2} \mathrm{O}\right) ;{ }^{1} \mathrm{H}$ NMR $\left(900 \mathrm{MHz}, \mathrm{D}_{2} \mathrm{O}\right) \delta 5.45\left(\mathrm{~d}, J=3.9 \mathrm{~Hz}, 1 \mathrm{H}, \mathrm{H}-1^{\prime}\right), 5.02(\mathrm{~d}, J=$ 3.7 Hz, 1H, H-1"), 4.14 (dd, $J=10.8,3.6$ Hz, 1H, H-2"), 3.95 (dd, $J=10.2,2.8$ Hz, 1H, H-5'), 3.93 (d, $\left.J=12.9 \mathrm{~Hz}, 1 \mathrm{H}, \mathrm{CH}_{2}, \mathrm{H}-5 \mathrm{a} "\right), 3.82-3.77$ (m, 2H, H-5, H-6), 3.73 - 3.67 (m, 2H, H-6', H-3'), $3.66-3.63$ (m, 1H, H-4), $3.61-3.54$ (m, 2H, H-2', H-1), 3.46 (ddd, J=12.9, 10.4, 4.2 Hz, 1H, H-3), 3.43 - 3.38 (m, 2H, H-3", CH2, H-5b"), 3.38 - 3.35 (m, 1H, H-4'), 2.83 (s, 3H), 2.46 (dt, J= 12.4, $4.3 \mathrm{~Hz}, 1 \mathrm{H}, \mathrm{H}-2 \mathrm{a}), 1.83$ (q, $J=12.5 \mathrm{~Hz}, 1 \mathrm{H}, \mathrm{H}-2 \mathrm{~b}), 1.26$ (s, 3H, $\mathrm{CH}_{3}$ ), 1.23 (d. $J=6.83 \mathrm{H}$, $\left.\mathrm{CH}_{3}\right) ;{ }^{13} \mathrm{C} \mathrm{NMR}\left(225 \mathrm{MHz}, \mathrm{D}_{2} \mathrm{O}\right) \delta 101.3,94.9,84.1,78.2,72.4,71.7,71.0,70.6,69.9,69.6,67.7$, 
66.3, 63.4, 49.8, 47.2, 47.0, 34.4, 27.5, 20.8, 10.9. ESI-HRMS: m/z calcd. for $\mathrm{C}_{20} \mathrm{H}_{41} \mathrm{O}_{10} \mathrm{~N} 4[\mathrm{M}+\mathrm{H}]^{+}$ 497.2823; found, 497.2806 .

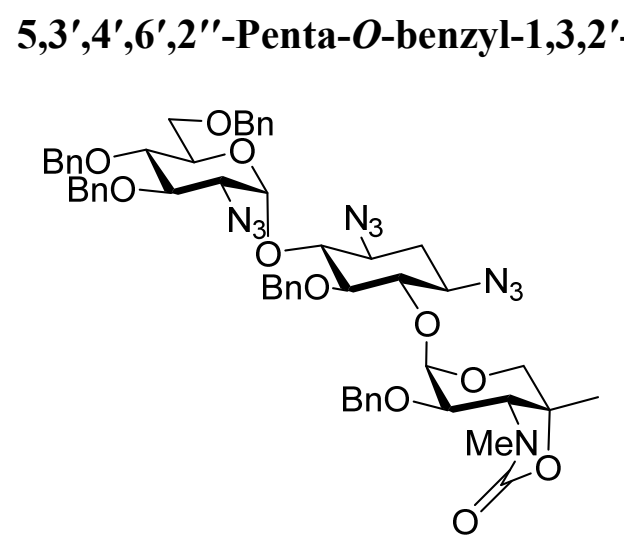

-triazido-1,3,2'-tri(deamino)-3"- $N, 4^{\prime \prime}$-O-carbonylgentamicin X2 (33). A mixture of thioglycoside $\mathbf{3 2}^{4}$ (111 mg; $0.19 \mathrm{mmol}$ ), and freshly activated $4 \AA$ acid-washed powdered molecular sieves were suspended in dichloromethane $(1.6 \mathrm{~mL})$. DMF (60 $\mu \mathrm{L}, 0.77 \mathrm{mmol})$ was added and the resulting mixture was stirred for $1 \mathrm{~h}$ at $\mathrm{rt}$ before it was cooled to $0{ }^{\circ} \mathrm{C}$ and stirred for $0.25 \mathrm{~h}$ before NIS (43 $\mathrm{mg}, 0.19 \mathrm{mmol})$ and TMSOTf $(35 \mu \mathrm{L}, 0.19 \mathrm{mmol})$ were added. After stirring for an additional $0.5 \mathrm{~h}$ at $0{ }^{\circ} \mathrm{C}$ a solution of glycosyl acceptor 23 ( $74 \mathrm{mg}, 0.13$ $\mathrm{mmol})$ in dichloromethane $(1.0 \mathrm{~mL})$ was added and the reaction mixture was slowly allowed to warm to $\mathrm{rt}$ and stirred for $24 \mathrm{~h}$ before it was quenched with triethylamine $(0.2 \mathrm{~mL})$. The mixture was diluted with dichloromethane, filtered through Celite ${ }^{\circledR}$, washed with $20 \%$ aqueous $\mathrm{Na}_{2} \mathrm{~S}_{2} \mathrm{O}_{3}$, dried over $\mathrm{Na}_{2} \mathrm{SO}_{4}$, and concentrated under reduced pressure. The crude product was purified using silica gel column chromatography (eluent: 20 - 30\% hexane/EtOAc) to give 33 (67 mg, 51\% and recovered $26(12 \mathrm{mg}, 16 \%)$, in the form of a colorless oil. $[\alpha]_{\mathrm{D}}{ }^{21}=+18.3\left(c 0.53, \mathrm{CHCl}_{3}\right) ;{ }^{1} \mathrm{H}$ NMR (600 MHz, $\left.\mathrm{CDCl}_{3}\right) \delta 7.53-7.19(\mathrm{~m}, 25 \mathrm{H}), 5.61(\mathrm{~d}, J=3.9 \mathrm{~Hz}, 1 \mathrm{H}, \mathrm{H}-1$ '), 5.20 (d, $J=3.5$ $\mathrm{Hz}, 1 \mathrm{H}, \mathrm{H}-1 "), 5.16$ (d, $J=10.9 \mathrm{~Hz}, 1 \mathrm{H}, \mathrm{PhCH} 2), 4.94-4.83$ (m, 3H, $\mathrm{PhCH}_{2}$ ), 4.79 (d, $J=10.9$ $\mathrm{Hz}, 2 \mathrm{H}, \mathrm{PhCH}_{2}$ ), 4.67 - 4.64 (m, 2H, $\mathrm{PhCH}_{2}$ ), $4.56-4.48$ (m, 2H, $\mathrm{PhCH}_{2}$ ), 4.20 (dt, J=10.1, 2.5 Hz, 1H, H-5"), 4.01 - 3.96 (m, 2H, H-2", H-3'), 3.82 (dd, J=10.8, 2.9 Hz, 1H, CH2, H-6'a), 3.78 3.73 (m, 3H, H-5, CH $2, \mathrm{H}-5$ "a, H-4'), $3.70-3.64$ (m, 2H, H-4, CH2, H-6'b), 3.52 (t, J=9.3 Hz, 1H, H-6), $3.51-3.44$ (m, 2H, H-1, H-3"), 3.41 (ddd, $J=12.5,9.9,4.6$ Hz, 1H, H-3), 3.36 (d, $J=12.6$ Hz, 1H, CH2, H-6'b), 3.28 (dd, $J=10.4,3.9$ Hz, 1H, H-2"), 2.82 (s, 3H, NCH3), 2.35 (dt, $J=13.1$, $4.5 \mathrm{~Hz}, 1 \mathrm{H}, \mathrm{H}-2 \mathrm{a}), 1.56-1.50$ (m, 1H, H-2b), 1.20 (s, 3H, $\left.\mathrm{CH}_{3}\right) ;{ }^{13} \mathrm{C} \mathrm{NMR}\left(151 \mathrm{MHz}, \mathrm{CDCl}_{3}\right) \delta$ 157.4, 138.2, 138.1, 137.9, 137.8, 137.4, 128.6, 128.4, 128.4, 128.4, 128.3, 128.2, 128.0, 128.0, $127.9,127.8,127.8,127.7,127.7,127.2,126.4,97.8,96.1,83.0,80.5,80.3,78.1,77.4,77.1,75.4$, 75.0, 74.9, 74.9, 73.6, 72.2, 71.3, 68.0, 66.9, 63.2, 63.1, 61.0, 59.2, 31.8, 30.0, 23.2; ESI-HRMS: $\mathrm{m} / \mathrm{z}$ calcd. for $\mathrm{C}_{55} \mathrm{H}_{60} \mathrm{O}_{11} \mathrm{~N}_{10} \mathrm{Na}[\mathrm{M}+\mathrm{Na}]^{+} 1059.4335$; found, 1059.4338 . 
Gentamicin X2 tetraaacetate salt (8). A suspension of compound $\mathbf{3 3}$ (23 mg, $21.9 \mu \mathrm{mol})$ and

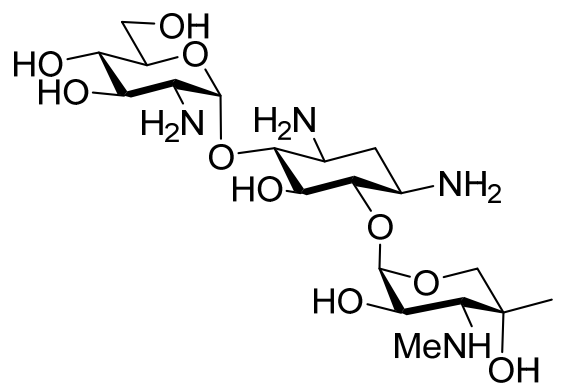
$\mathrm{Pd}(\mathrm{OH})_{2} / \mathrm{C}(10 \%, 50 \mathrm{mg})$ in dioxane: $\mathrm{H}_{2} \mathrm{O}(1: 1,0.8 \mathrm{~mL})$ was subjected to hydrogenolysis under 40 psi of $\mathrm{H}_{2}$ for $36 \mathrm{~h}$. After completion of the reaction, the catalyst was filtered off and the filtrate was concentrated to dryness. The crude residue was re-dissolved in a saturated aqueous solution of $\mathrm{Ba}(\mathrm{OH})_{2}$ $(0.5 \mathrm{~mL})$ and heated to $60{ }^{\circ} \mathrm{C}$ for $12 \mathrm{~h}$. After complete hydrolysis of the oxazolidinone, the reaction mixture was neutralized to $\mathrm{pH} 7$ by addition of dry ice and the precipitated solid was filtered off. Th filter cake was washed with water and the filtrate was concentrated to dryness. The crude product was taken up in 10\% aqueous acetic acid and loaded on a prepacked Sephadex C-25 column and eluted with $0.1 \%$ to $1.5 \%$ ammonium hydroxide in deionized water. The product-containing fractions were combined, concentrated, acidified with glacial acetic acid, and lyophilized to give the pentaacetate salt of gentamicin X2 (8) as a white foam (6.6 mg, $61 \%)$. [ $\alpha]_{\mathrm{D}^{21}}=+64.2\left(c 0.07, \mathrm{H}_{2} \mathrm{O}\right)$; $1 \mathrm{H} \mathrm{NMR}\left(600 \mathrm{MHz}, \mathrm{D}_{2} \mathrm{O}\right) \delta 5.60(\mathrm{~d}, J=4.1$ Hz, 1H, H-1'), 5.04 (d, J=3.7 Hz, 1H, H-1"), 4.17 (dd, J=10.9, 3.7 Hz, 1H, H-2"), 3.94 (d, $J=$ $\left.12.8 \mathrm{~Hz}, 1 \mathrm{H}, \mathrm{CH}_{2}, \mathrm{H}-5 \mathrm{a}\right)$ ), 3.90 - 3.79 (m, 3H), 3.80 - 3.66 (m, 4H), $3.54-3.38$ (m, 6H), 2.86 (s, $3 \mathrm{H}, \mathrm{NCH}_{3}$ ), 2.48 (dt, $\left.J=12.7,4.3 \mathrm{~Hz}, 1 \mathrm{H}, \mathrm{H}-2 \mathrm{a}\right), 1.85$ (q, $\left.J=12.6 \mathrm{~Hz}, 1 \mathrm{H}, \mathrm{H}-2 \mathrm{~b}\right), 1.29$ (s, 3H, $\left.\mathrm{CH}_{3}\right) .{ }^{13} \mathrm{C} \mathrm{NMR}\left(151 \mathrm{MHz}, \mathrm{D}_{2} \mathrm{O}\right) \delta 101.2,97.2,83.5,80.6,73.8,73.7,69.8,69.3,69.0,67.8,66.2$, 63.3, 60.4, 53.9, 49.5, 48.6, 34.5, 27.9, 20.9; ESI-HRMS: m/z calcd. for $\mathrm{C}_{19} \mathrm{H}_{39} \mathrm{O}_{10} \mathrm{~N}_{4}[\mathrm{M}+\mathrm{H}]^{+}$ 483.2666; found, 483.2649. 


\section{References}

1. Sasaki, E.; Lin, C.-I.; Lin, K.-Y.; Liu, H.-w., Construction of the Octose 8-Phosphate Intermediate in Lincomycin a Biosynthesis: Characterization of the Reactions Catalyzed by Lmbr and Lmbn J. Am. Chem. Soc. 2012, 134, 17432-17435.

2. Spohr, U.; Le, N.; Ling, C.-C.; Lemieux, R. U., The Syntheses of 6-C-Alkyl Derivatives of Methyl A-Isomaltoside for a Study of the Mechanism of Hydrolysis by Amyloglucosidase. Can. J. Chem. 2001, 79, 238-255.

3. Hanessian, S.; Maianti, J. P., Biomimetic Synthesis and Structural Refinement of the Macrocyclic Dimer Aminoglycoside 66-40c-The Remarkably Selective SelfCondensation of a Putative Aldehyde Intermediate in the Submerged Culture Medium Producing Sisomicin. Chem. Commun. 2010, 46, 2013-2015.

4. Chang, C.-W.; Wu, C.-H.; Lin, M.-H.; Liao, P.-H.; Chang, C.-C.; Chuang, H.-H.; Lin, S.-C.; Lam, S.; Verma, V. P.; Hsu, C.-P.; Wang, C.-C., Establishment of Guidelines for the Control of Glycosylation Reactions and Intermediates by Quantitative Assessment of Reactivity Angew. Chem. Int. Ed. 2019, 58, 16775-16779. 

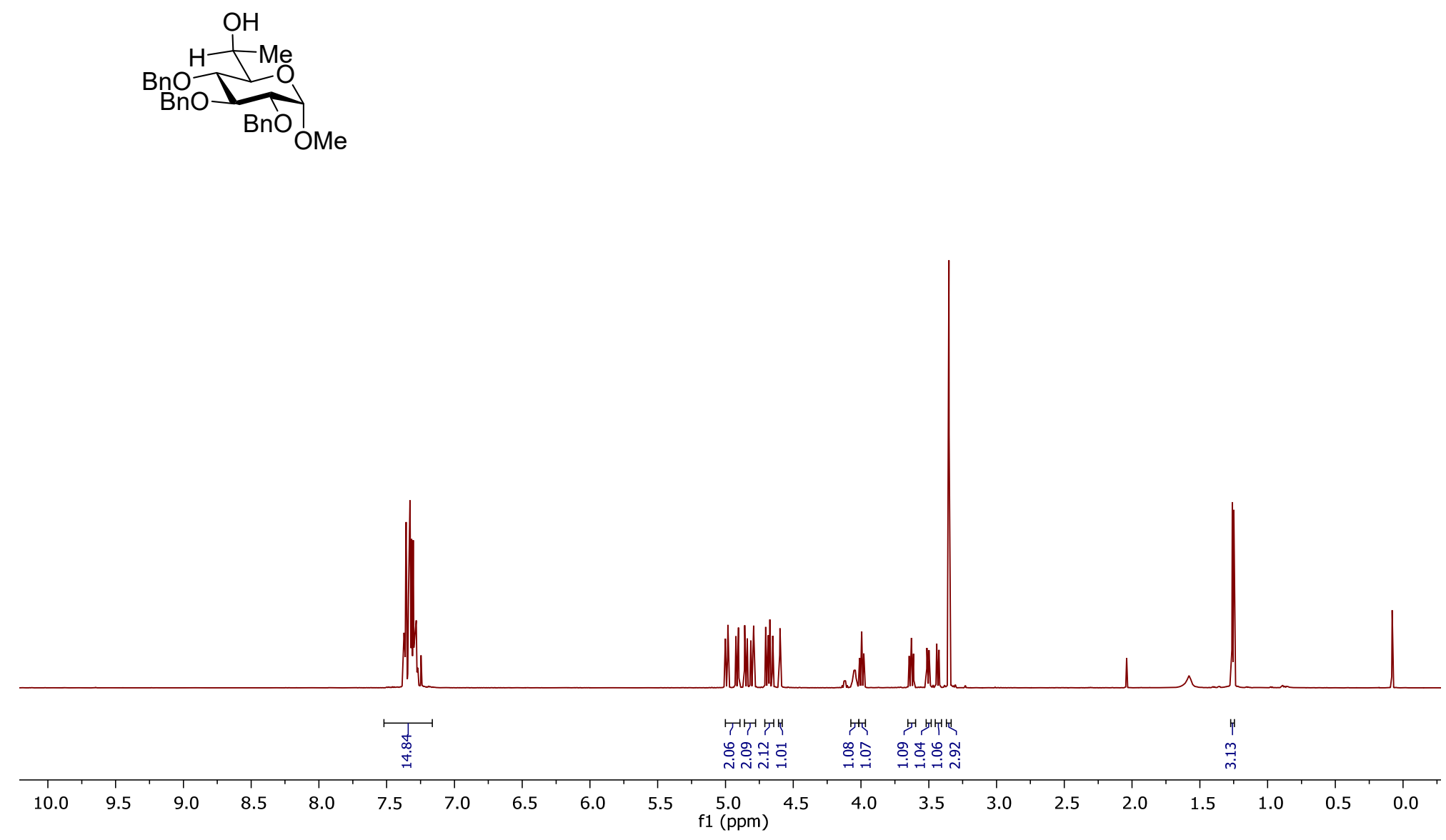

${ }^{1} \mathrm{H}$ NMR (600 MHz, $\mathrm{CDCl}_{3}$ ) of Methyl 2,3,4-tri-O-benzyl-7-deoxy- $\alpha$-L-glycero-D-gluco-heptopyranoside (28) 

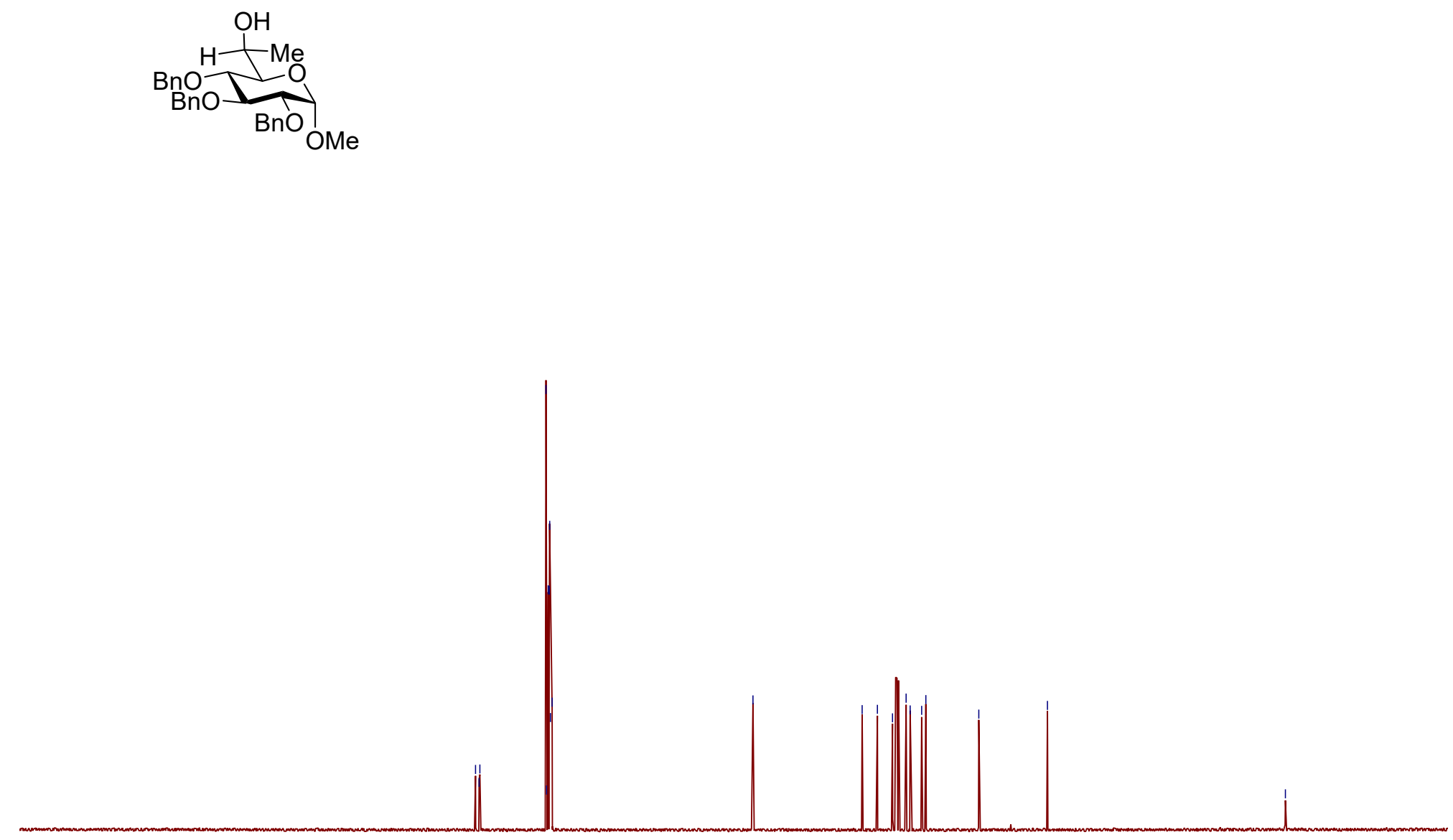

$\begin{array}{llllllll}200 & 190 & 180 & 170 & 160 & 150 & 140 & 130\end{array}$ 120 $110 \quad 100$ $90 \quad 80$ $70 \quad 60$ $60 \quad 50$

${ }^{13} \mathrm{C}$ NMR (150 MHz, $\mathrm{CDCl}_{3}$ ) of Methyl 2,3,4-tri-O-benzyl-7-deoxy- $\alpha$-L-glycero-D-gluco-heptopyranoside (28) 

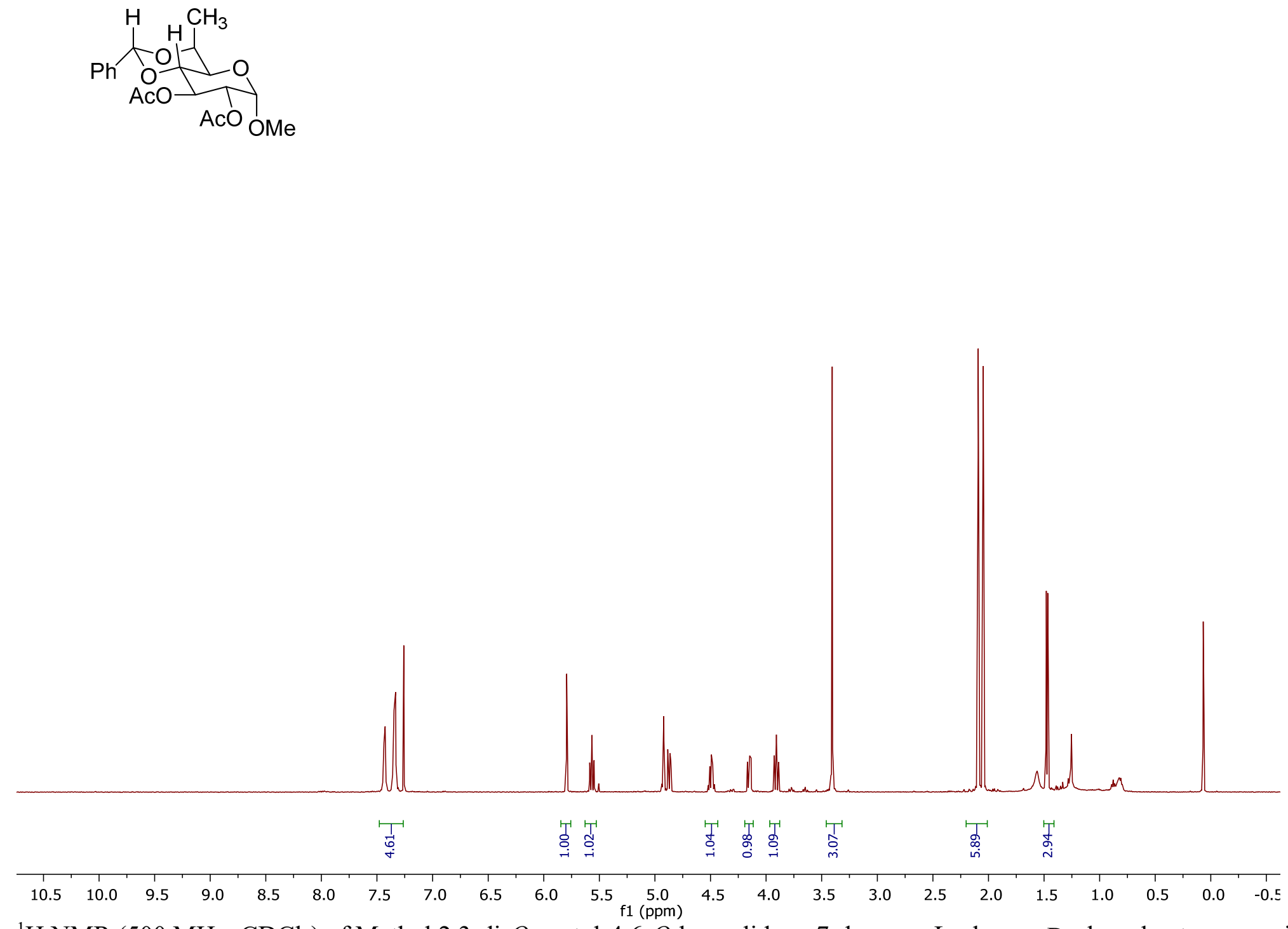

${ }^{1} \mathrm{H}$ NMR $\left(500 \mathrm{MHz}, \mathrm{CDCl}_{3}\right.$ ) of Methyl 2,3-di-O-acetyl-4,6-O-benzylidene-7-deoxy- $\alpha$-L-glycero-D-gluco-heptopyranoside (S1) 


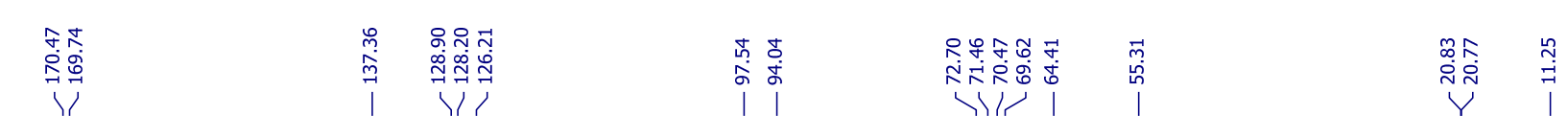
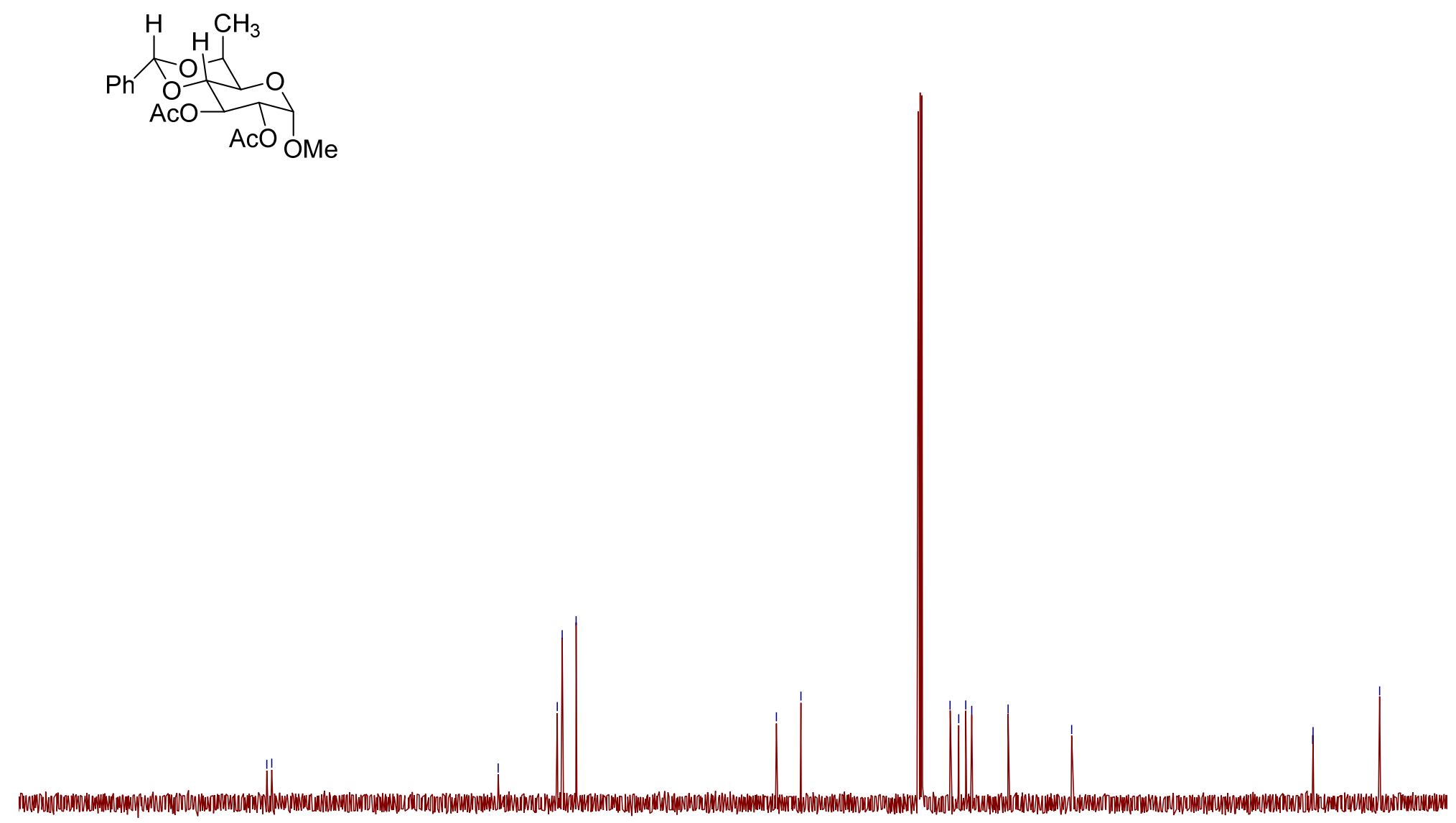

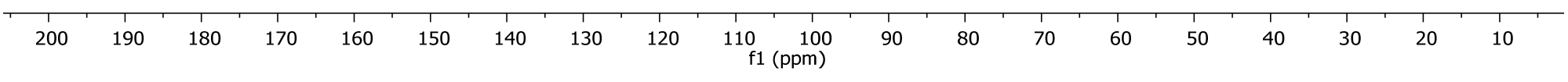

${ }^{13} \mathrm{C}$ NMR (125 MHz, $\mathrm{CDCl}_{3}$ ) of Methyl 2,3-di- $O$-acetyl-4,6-O-benzylidene-7-deoxy- $\alpha$-L-glycero-D-gluco-heptopyranoside (S1). 

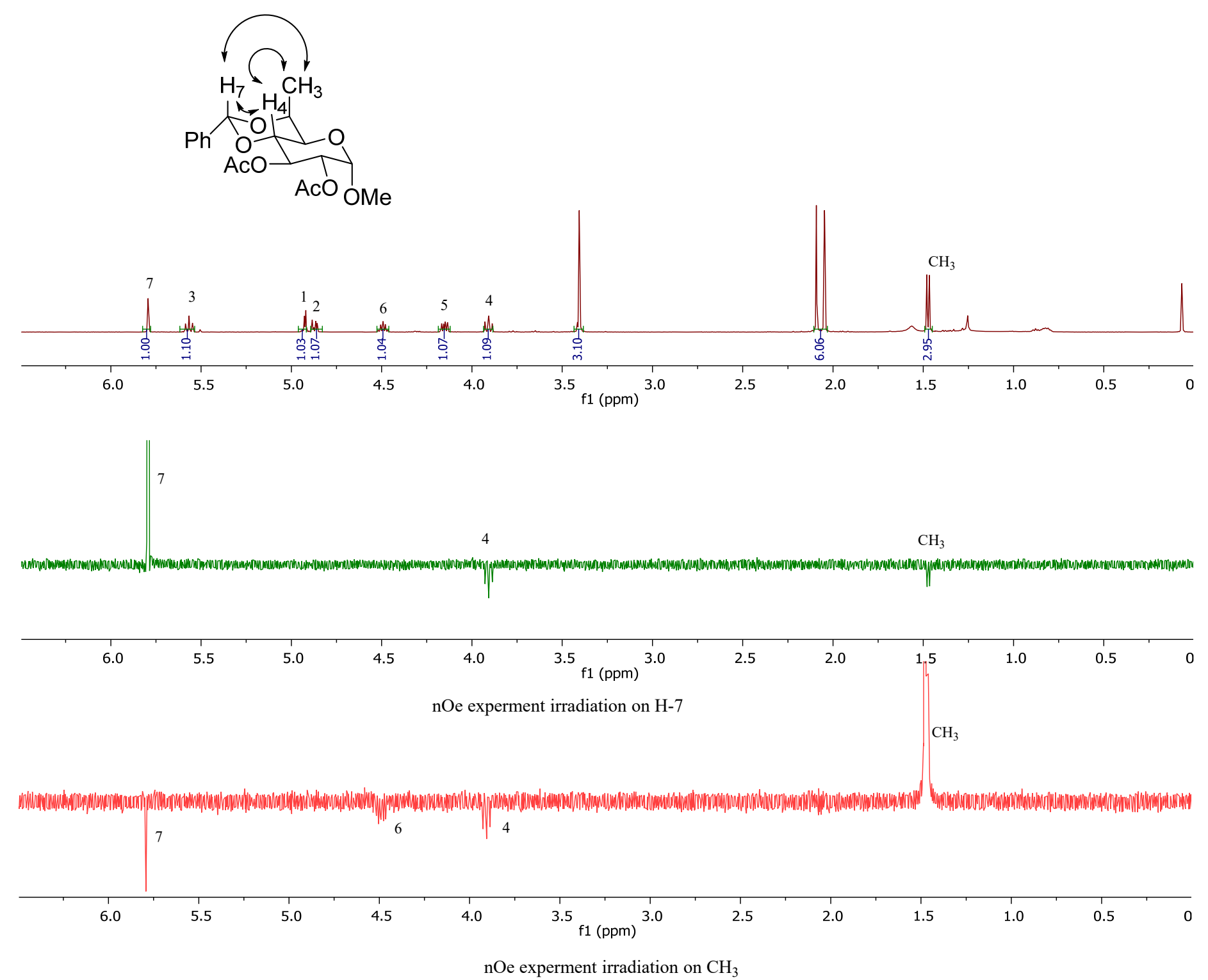

nOe Spectra (500 MHz, $\mathrm{CDCl}_{3}$ ) of Methyl 2,3-di-O-acetyl-4,6-O-benzylidene-7-deoxy- $\alpha$-L-glycero-D-gluco-heptopyranoside (S1) 

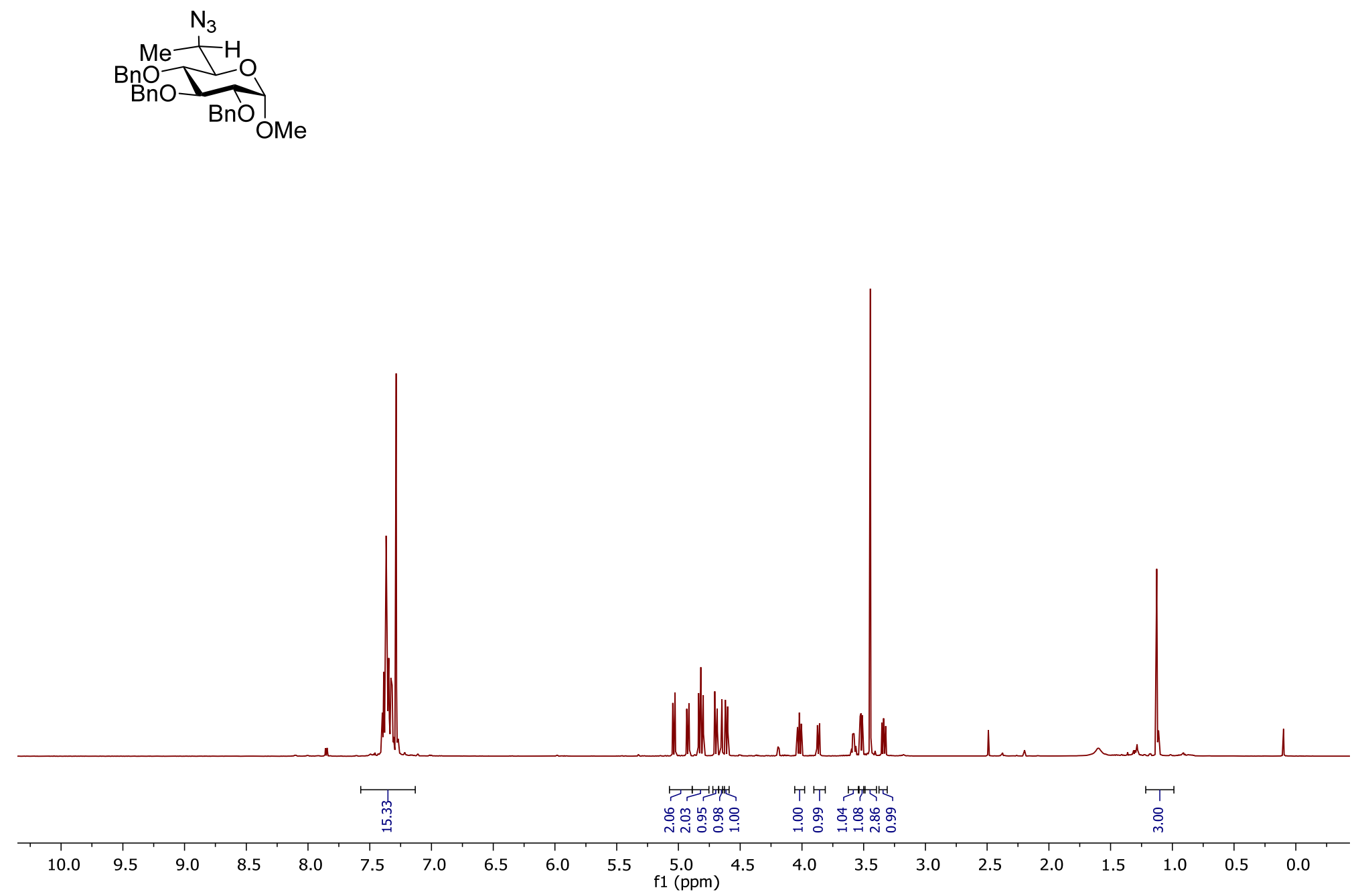

${ }^{1} \mathrm{H}$ NMR (600 MHz, CDCl 3 ) of Methyl 6-azido-2,3,4-tri-O-benzyl-6,7-dideoxy- $\alpha$-D-glycero-D-gluco-heptopyranoside (29). 


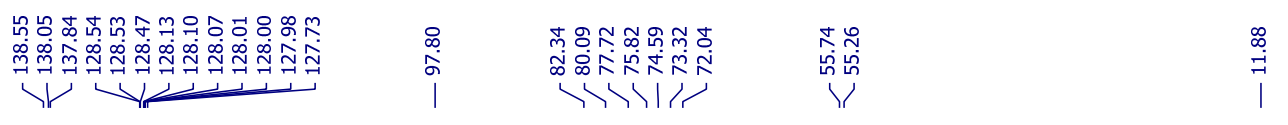
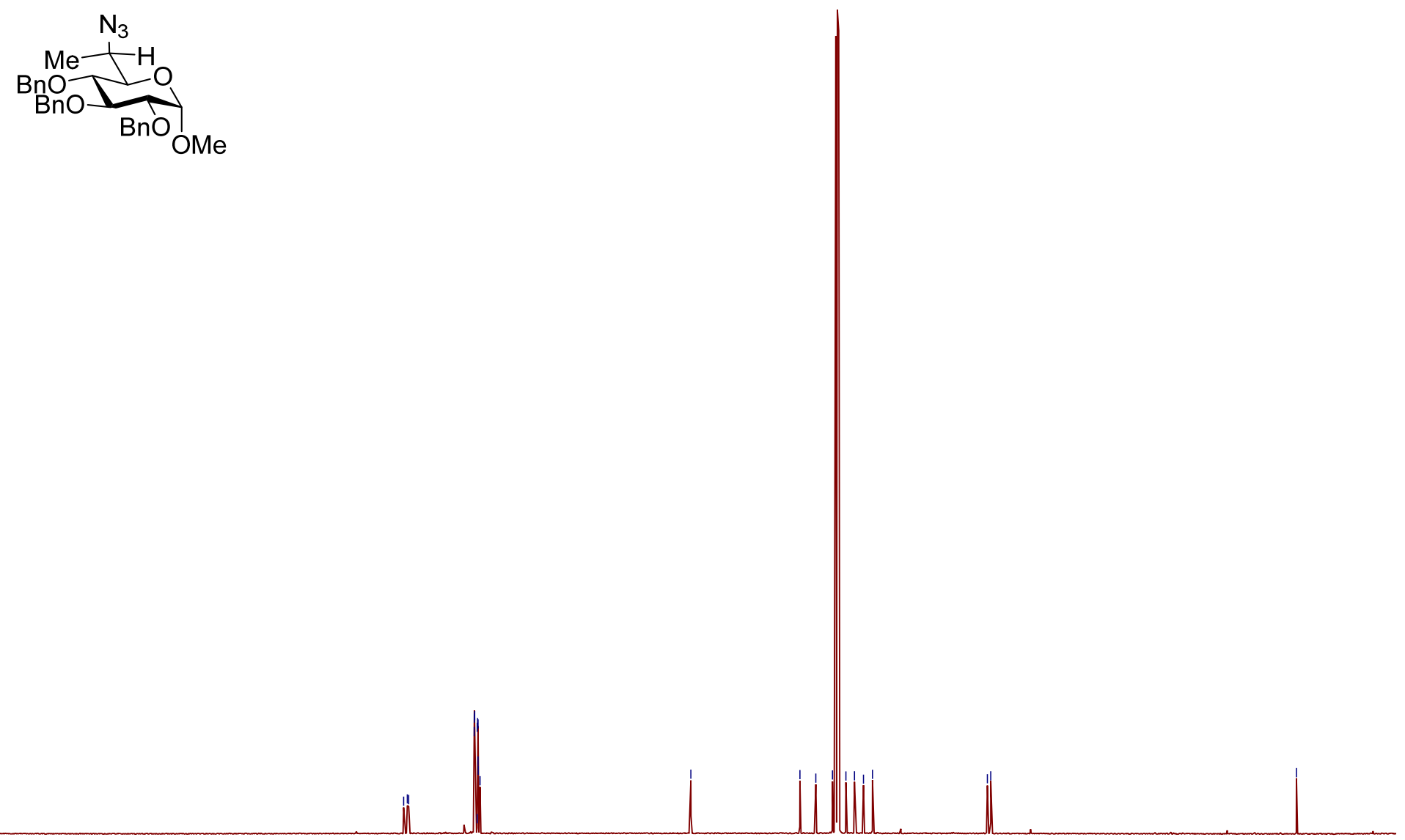

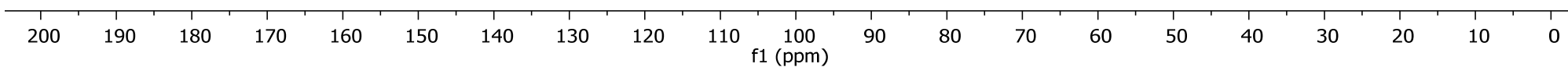

${ }^{13} \mathrm{C}$ NMR (150 MHz, $\mathrm{CDCl}_{3}$ ) of Methyl 6-azido-2,3,4-tri-O-benzyl-6,7-dideoxy- $\alpha$-D-glycero-D-gluco-heptopyranoside (29). 


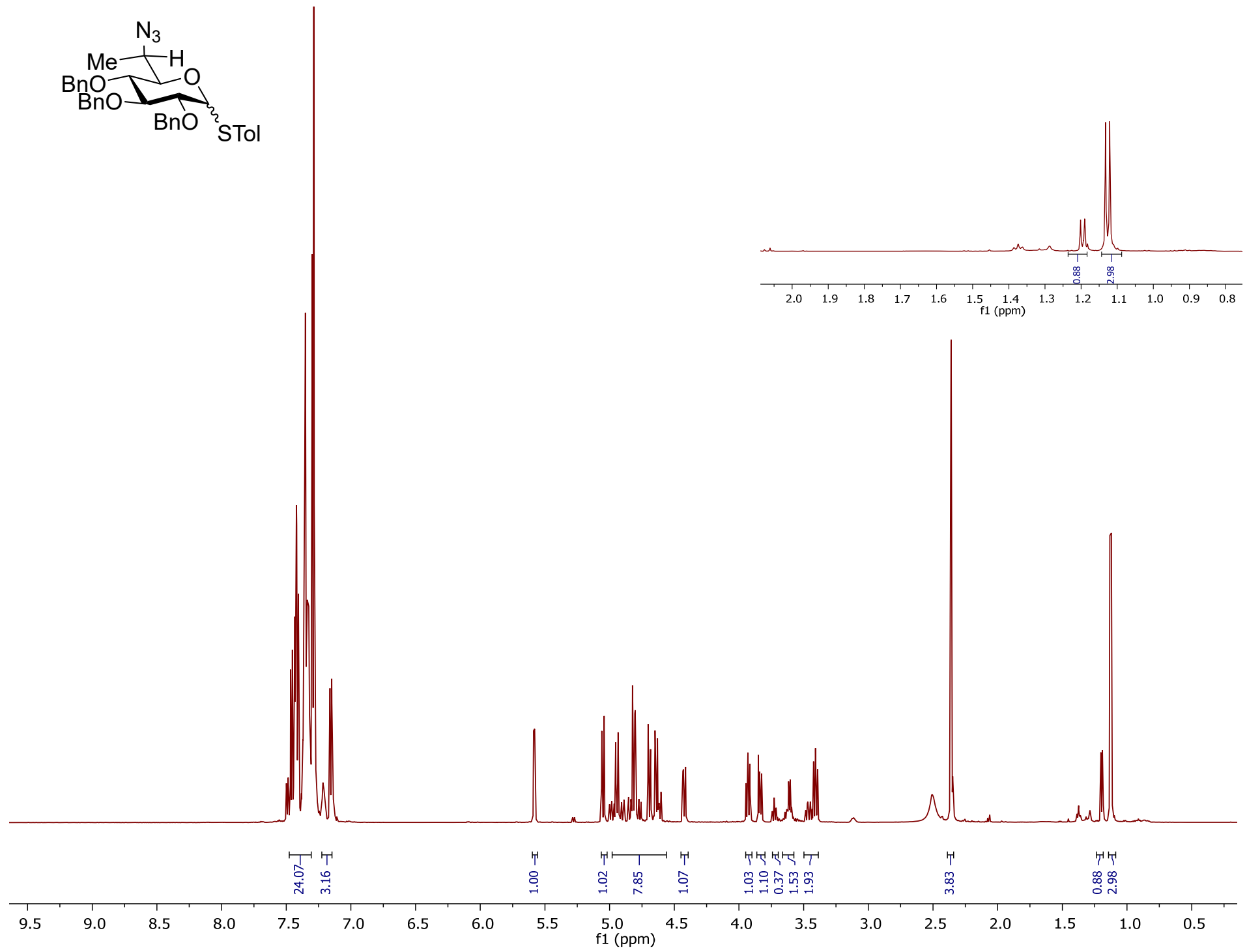

${ }^{1} \mathrm{H}$ NMR $\left(600 \mathrm{MHz}, \mathrm{CDCl}_{3}\right.$ ) of $p$-Tolyl 6-azido-2,3,4-tri-O-benzyl-6,7-dideoxy- $\alpha, \beta$-D-glycero-D-gluco-heptothiopyranoside (30).

S20 

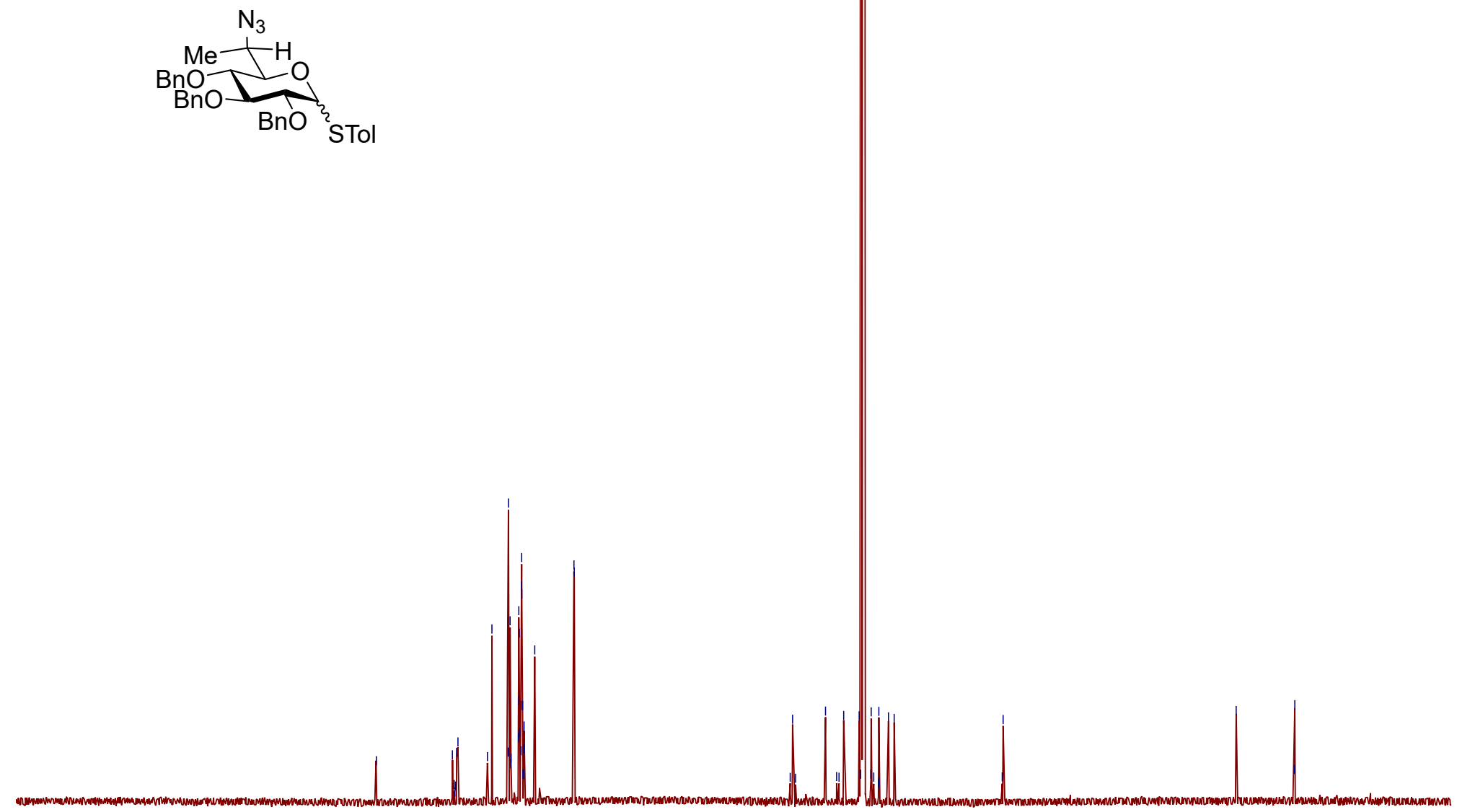

200 $\begin{array}{lllllllll}180 & 170 & 160 & 150 & 140 & 130 & 120 & 110 & 100 \\ \mathrm{f} 1(\mathrm{ppm})\end{array}$

${ }^{13} \mathrm{C}$ NMR $\left(150 \mathrm{MHz}, \mathrm{CDCl}_{3}\right.$ ) of $p$-Tolyl 6-azido-2,3,4-tri-O-benzyl-6,7-dideoxy- $\alpha, \beta$-D-glycero-D-gluco-heptothiopyranoside (30). 


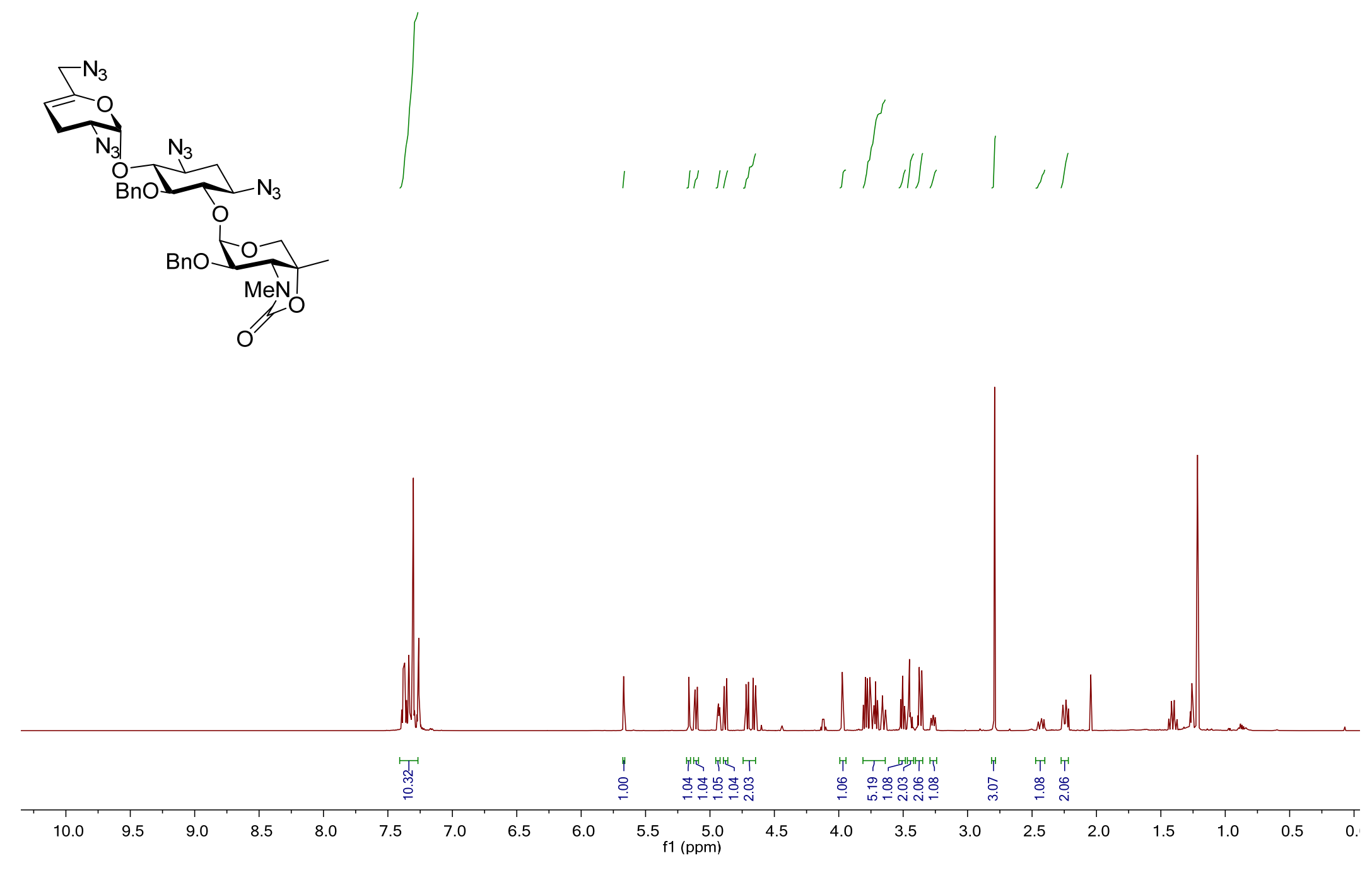

${ }^{1} \mathrm{H}$ NMR (600 MHz, CDCl 3 ) 1,3,2',6'-Tetra(deamino)-5,2"'-di-O-benzyl-1,3,2',6'-tetraazido-3"'-N,4"'-O-carbonyl-sisomycin (25). 

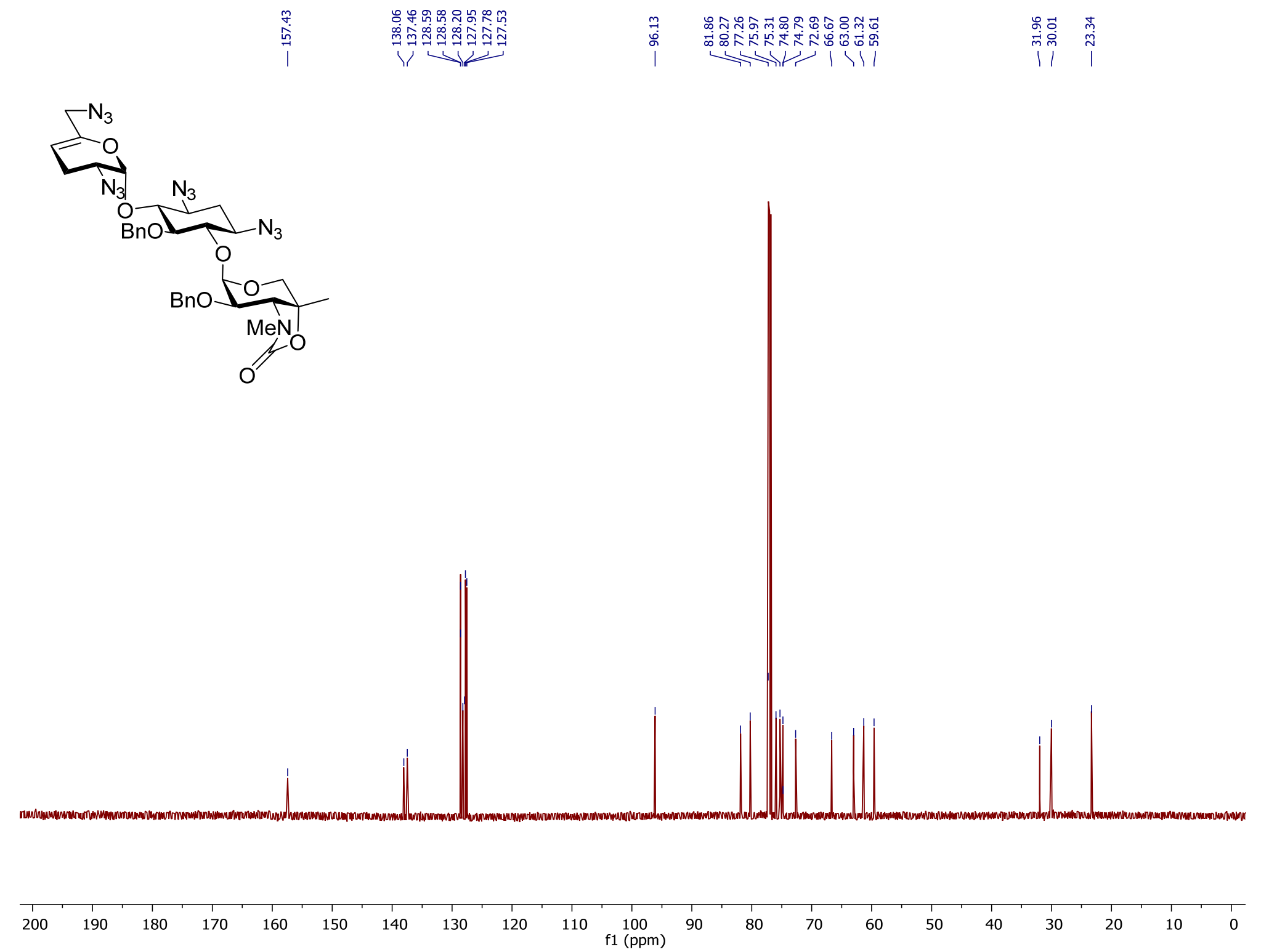

${ }^{13} \mathrm{C}$ NMR (150 MHz, CDCl 3 ) 1,3,2',6'-Tetra(deamino)-5,2"-di-O-benzyl-1,3,2',6'-tetraazido-3"'- $N, 4^{\prime \prime}$ - $O$-carbonyl-sisomycin (25). 

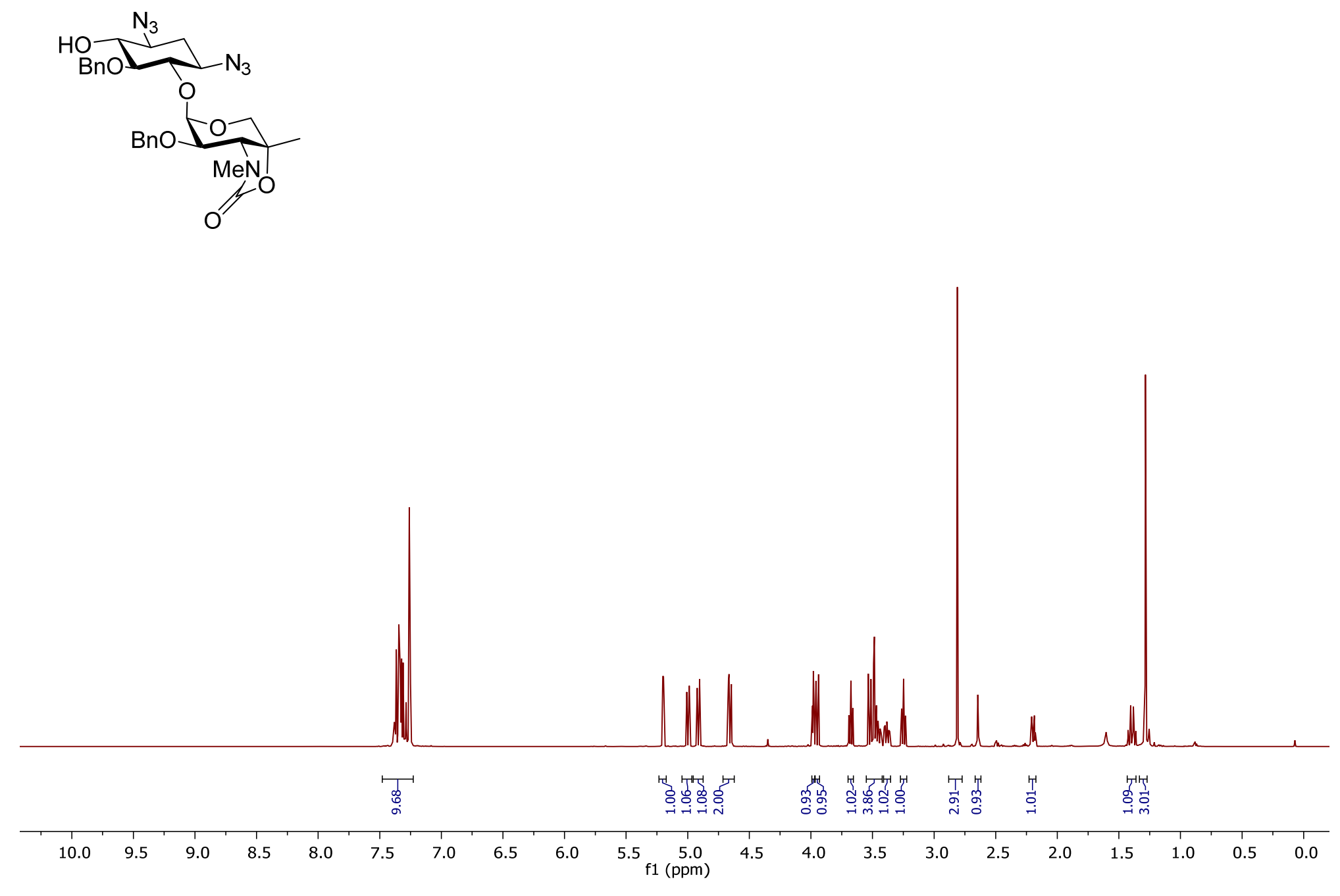

${ }^{1} \mathrm{H}$ NMR (600 MHz, $\mathrm{CDCl}_{3}$ ) 5,2'-Di- $O$-benzyl-1,3-di(deamino)-1,3-diazido-3'- $N, 44^{\prime}-O$-carbonyl-garamine (26). 

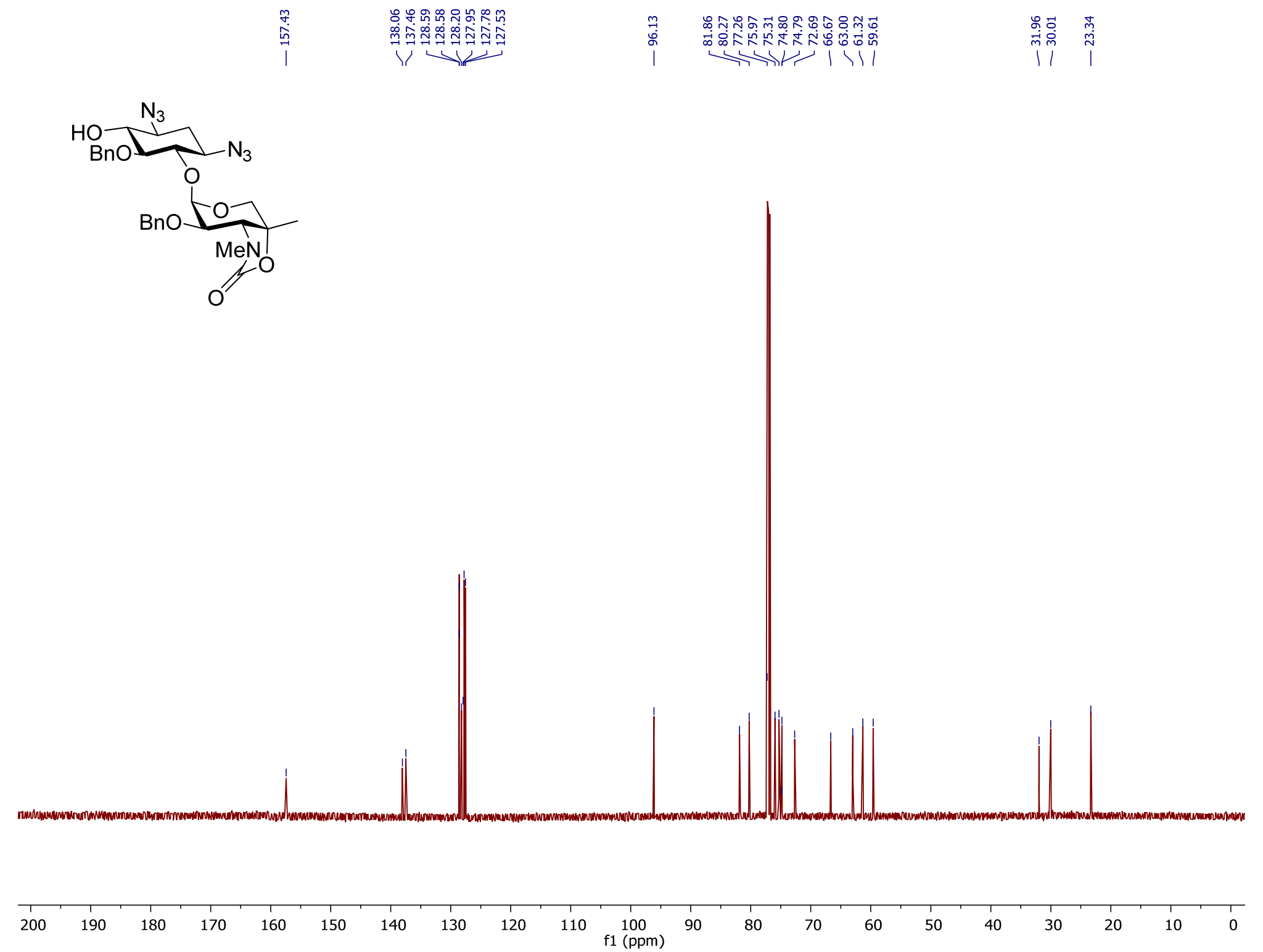

${ }^{13} \mathrm{C}$ NMR (150 MHz, $\mathrm{CDCl}_{3}$ ) 5,2'-Di-O-benzyl-1,3-di(deamino)-1,3-diazido-3'- $N, 4^{\prime}$-O-carbonyl-garamine (26). 


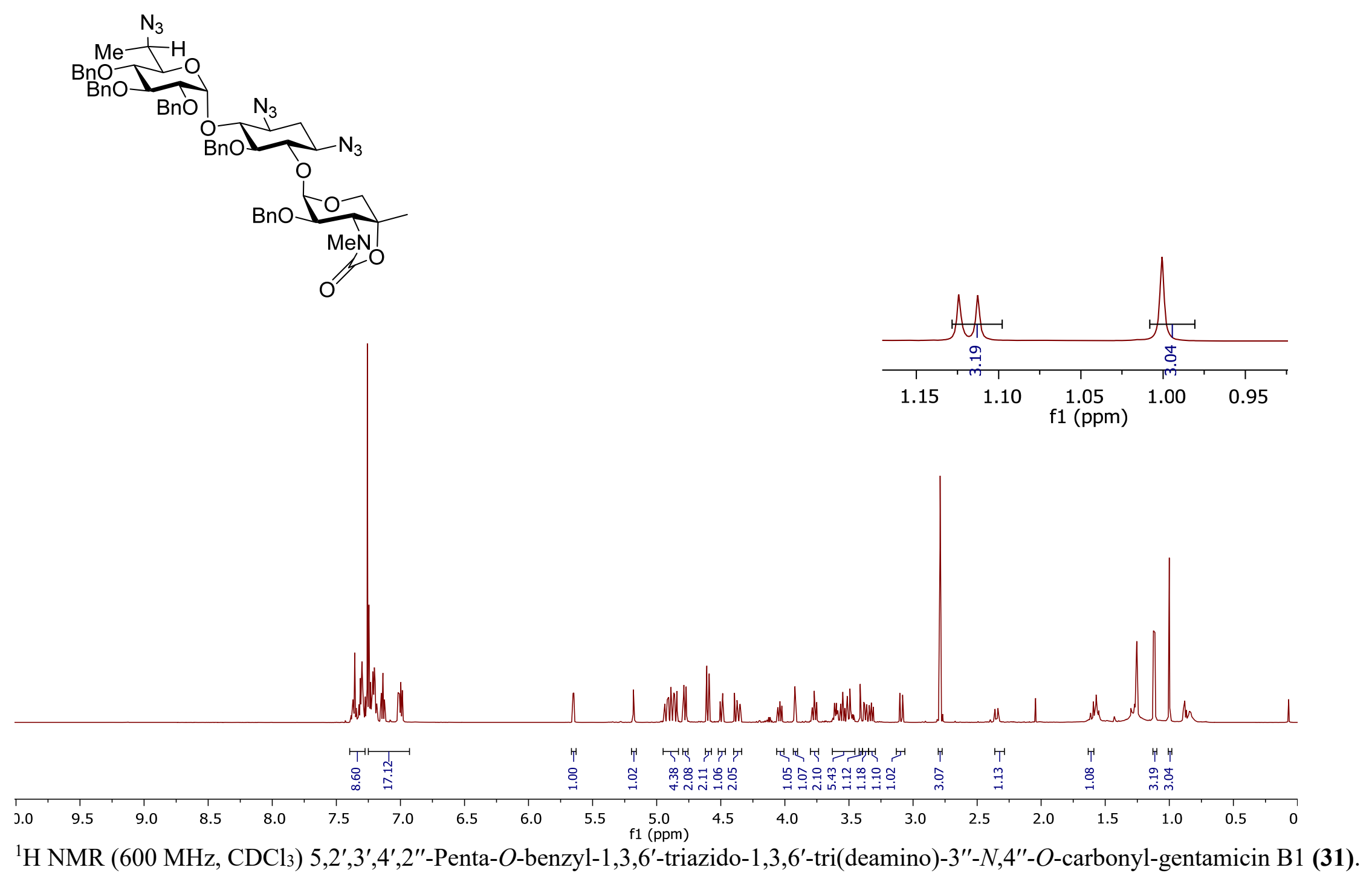




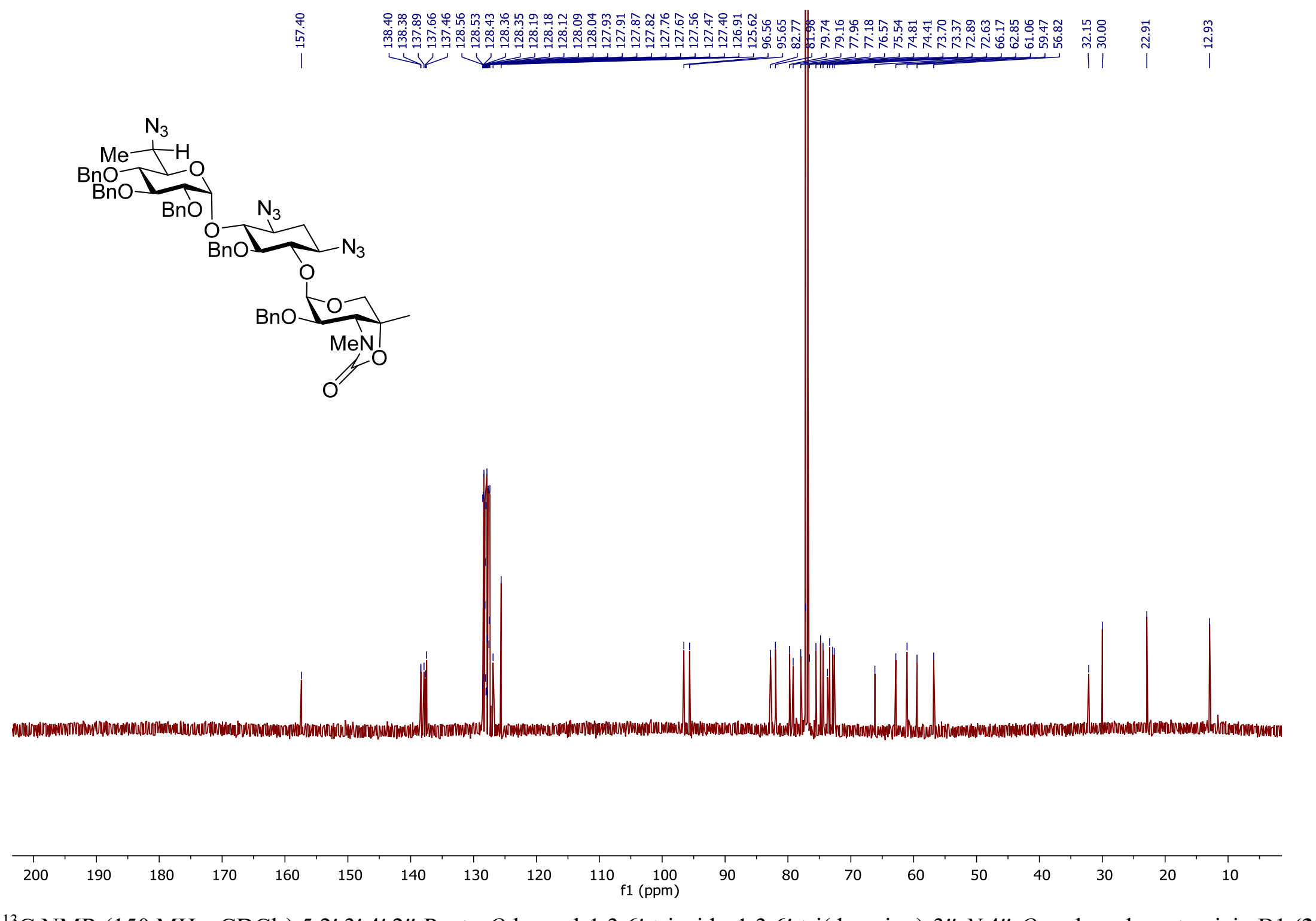

${ }^{13} \mathrm{C}$ NMR (150 MHz, CDCl 3 ) 5,2',3',4',2"-Penta-O-benzyl-1,3,6'-triazido-1,3,6'-tri(deamino)-3"'-N,4"-O-carbonyl-gentamicin B1 (31). 


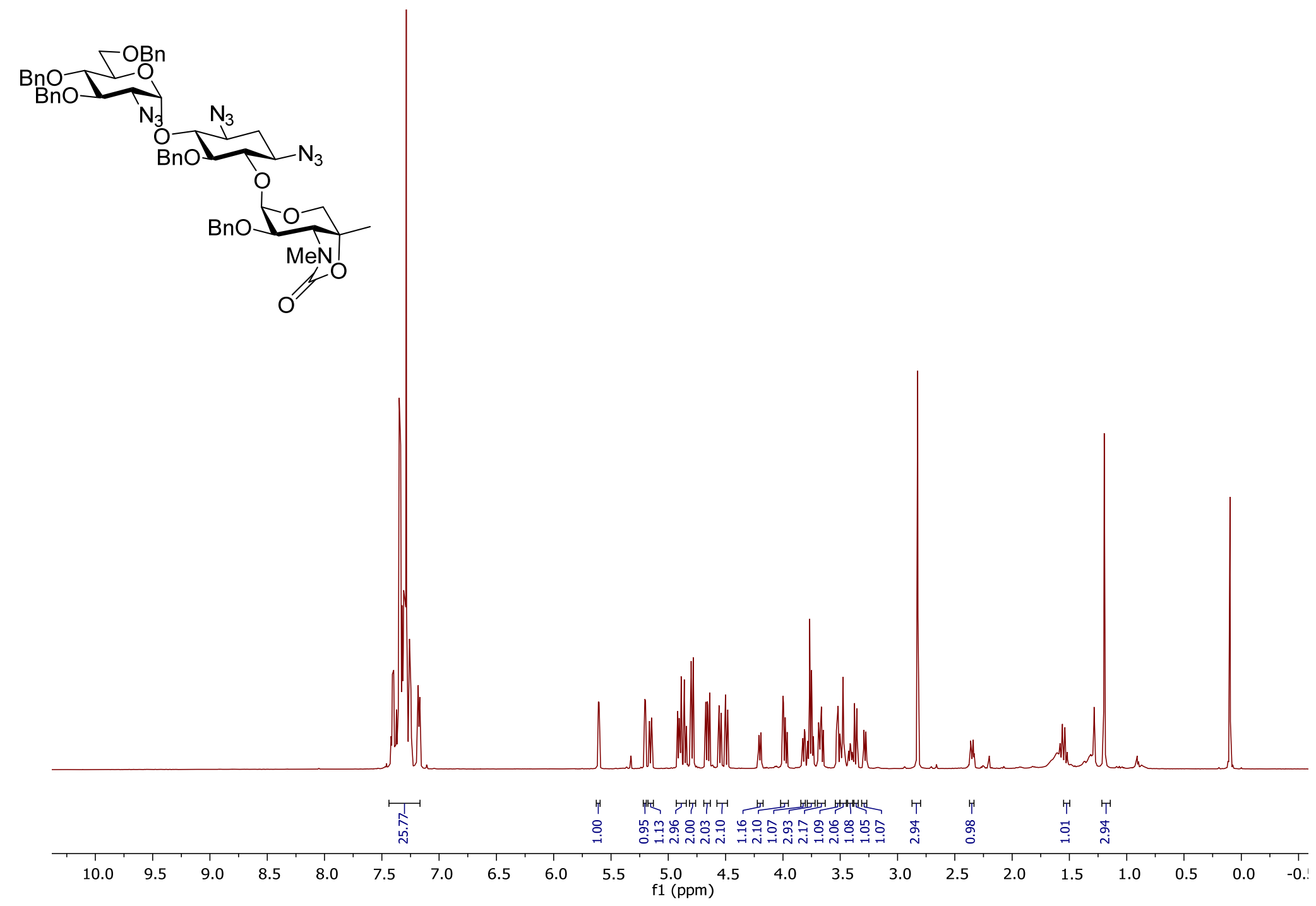

${ }^{1} \mathrm{H}$ NMR $\left(600 \mathrm{MHz}, \mathrm{CDCl}_{3}\right)$ of 5,3',4',6',2'-Penta-O-benzyl-1,3,2'-triazido-1,3,2'-tri(deamino)-3"'-N,4"-O-carbonyl-gentamicin X2 (33). 


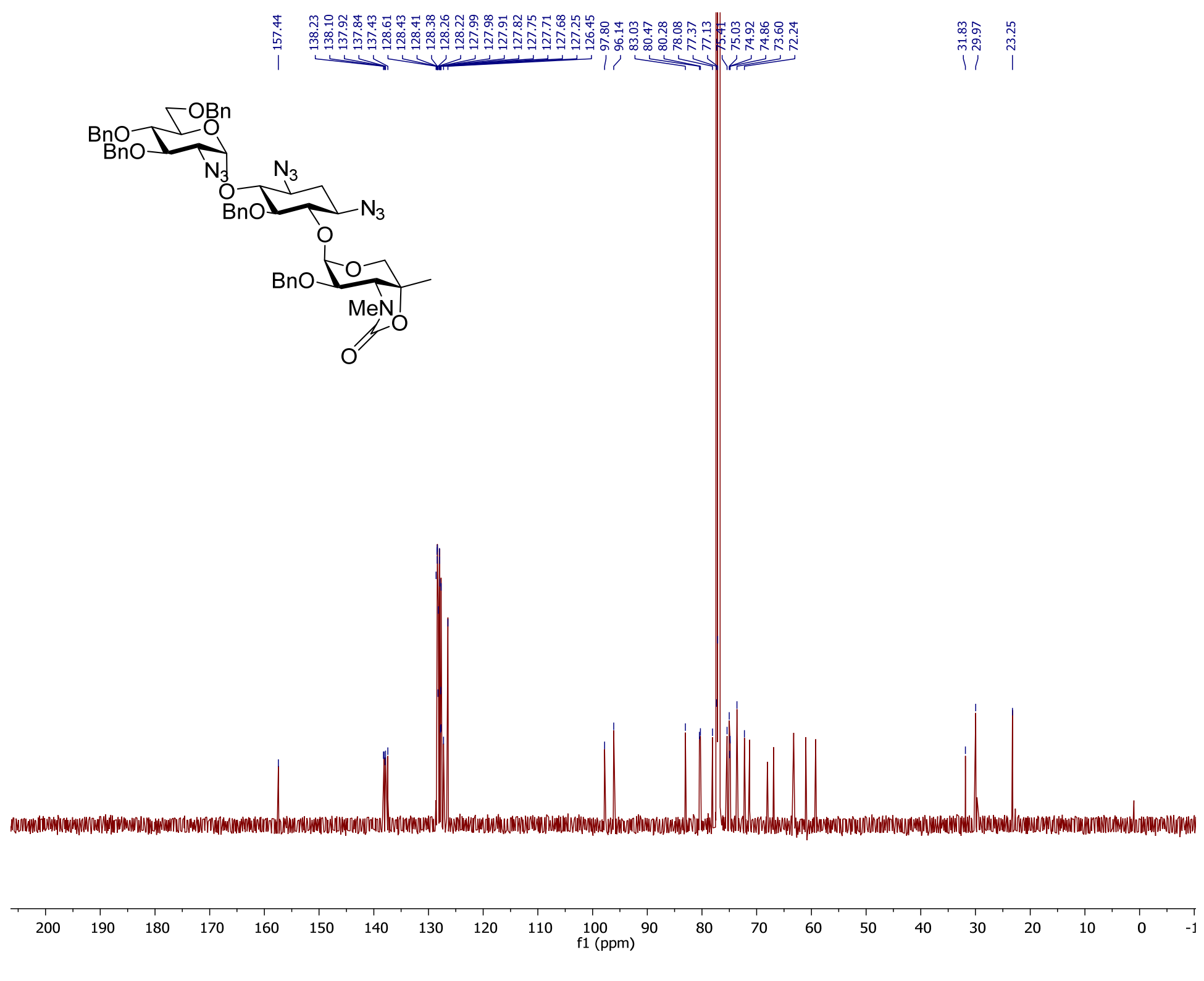

${ }^{13} \mathrm{C}$ NMR (150 MHz, $\left.\mathrm{CDCl}_{3}\right)$ of 5,3',4',6',2'-Penta-O-benzyl-1,3,2'-triazido-1,3,2'-tri(deamino)-3"-N,4"-O-carbonyl-gentamicin X2 (33). 


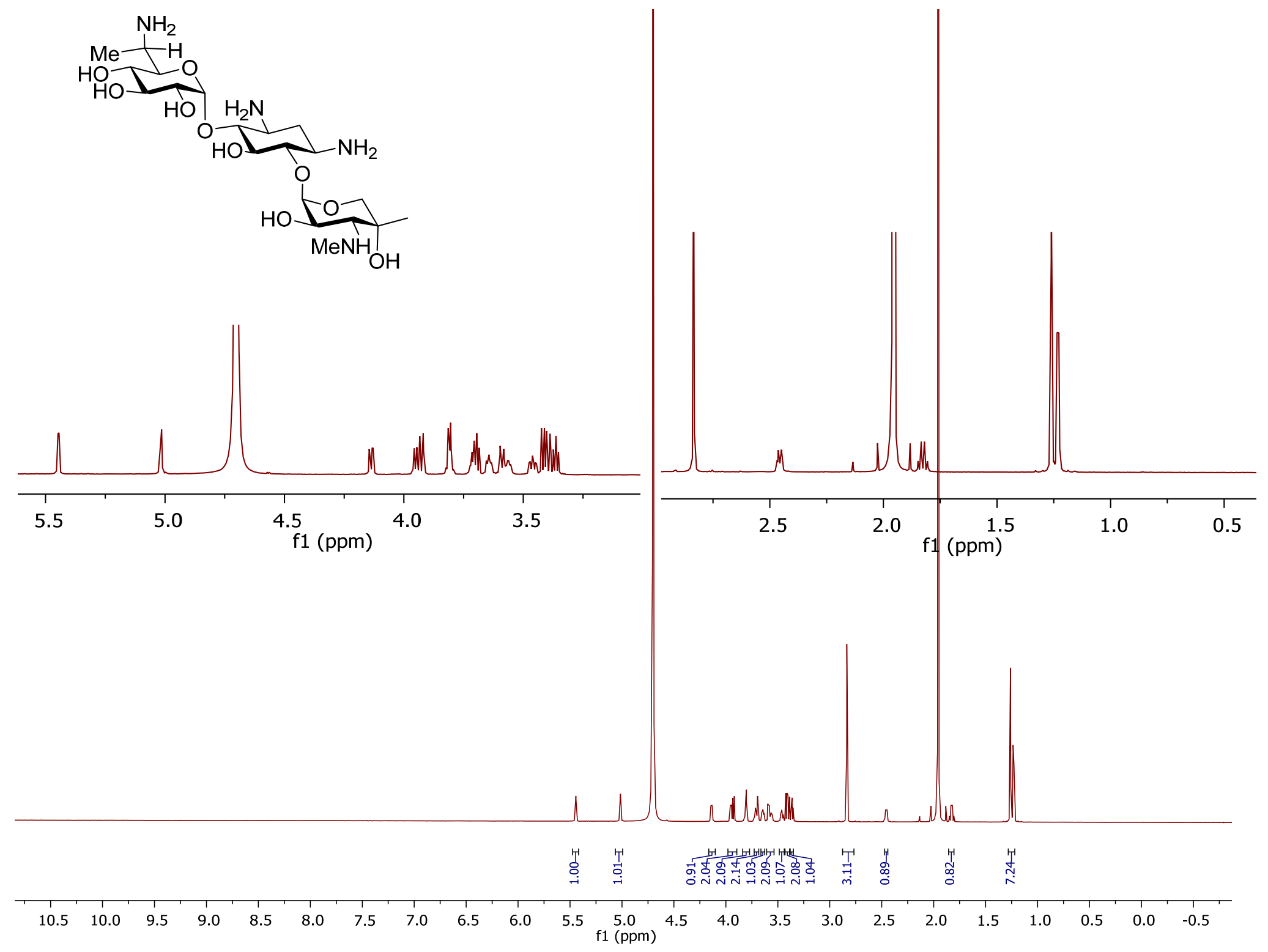

${ }^{1} \mathrm{H}$ NMR (900 MHz, D2O) of Gentamicin B1 tetraaacetate salt (7). 


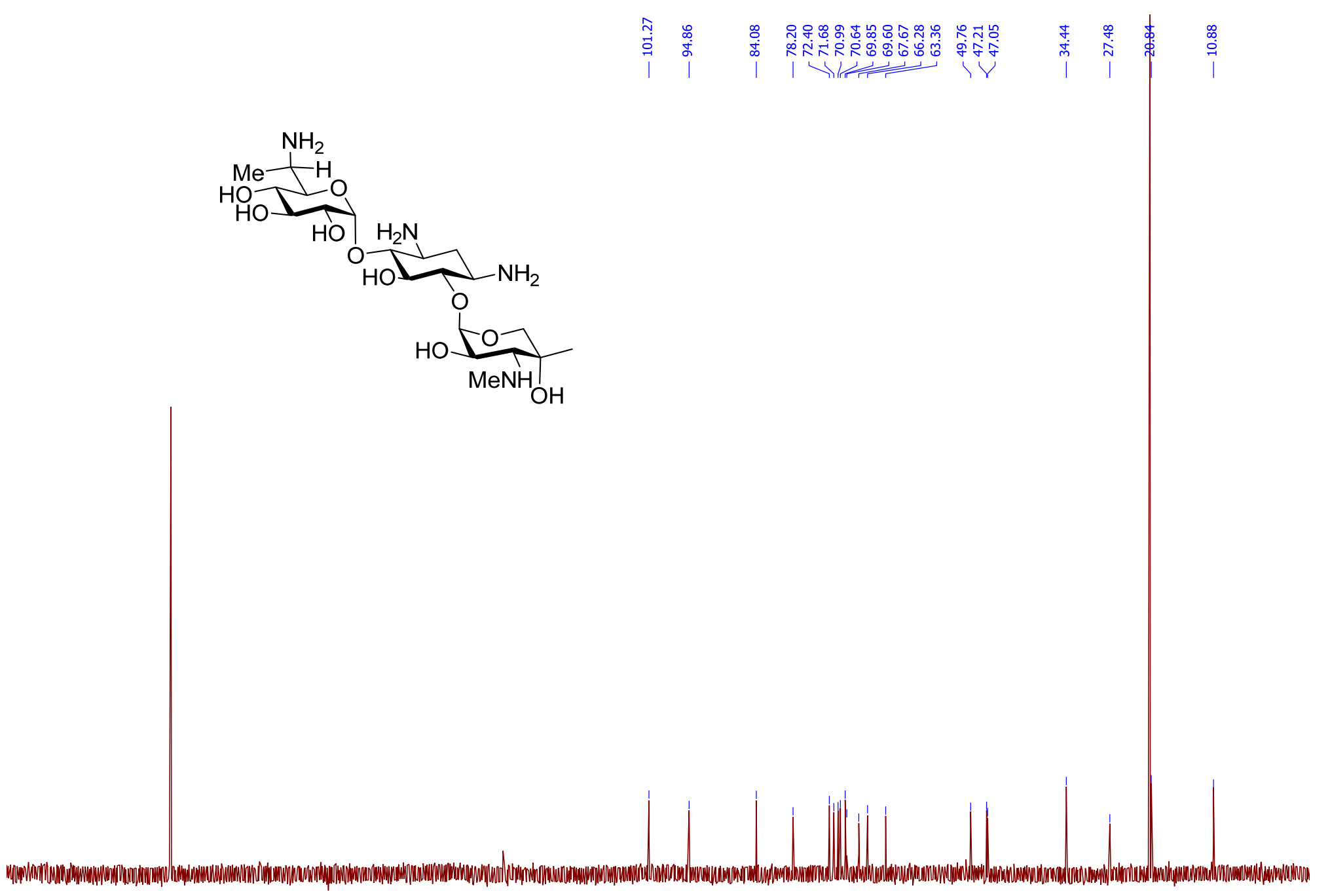

\begin{tabular}{|c|c|c|c|c|c|c|c|c|c|c|c|c|c|c|c|c|c|c|c|c|}
\hline 200 & 190 & 180 & 170 & 160 & 150 & 140 & 130 & 120 & 110 & $\begin{array}{c}100 \\
\mathrm{f} 1(\mathrm{ppm})\end{array}$ & 90 & 80 & 70 & 60 & 50 & 40 & 30 & 20 & 10 & 0 \\
\hline
\end{tabular}

${ }^{13} \mathrm{C}$ NMR (225 MHz, $\mathrm{D}_{2} \mathrm{O}$ ) of Gentamicin B1 tetraaacetate salt (7). 


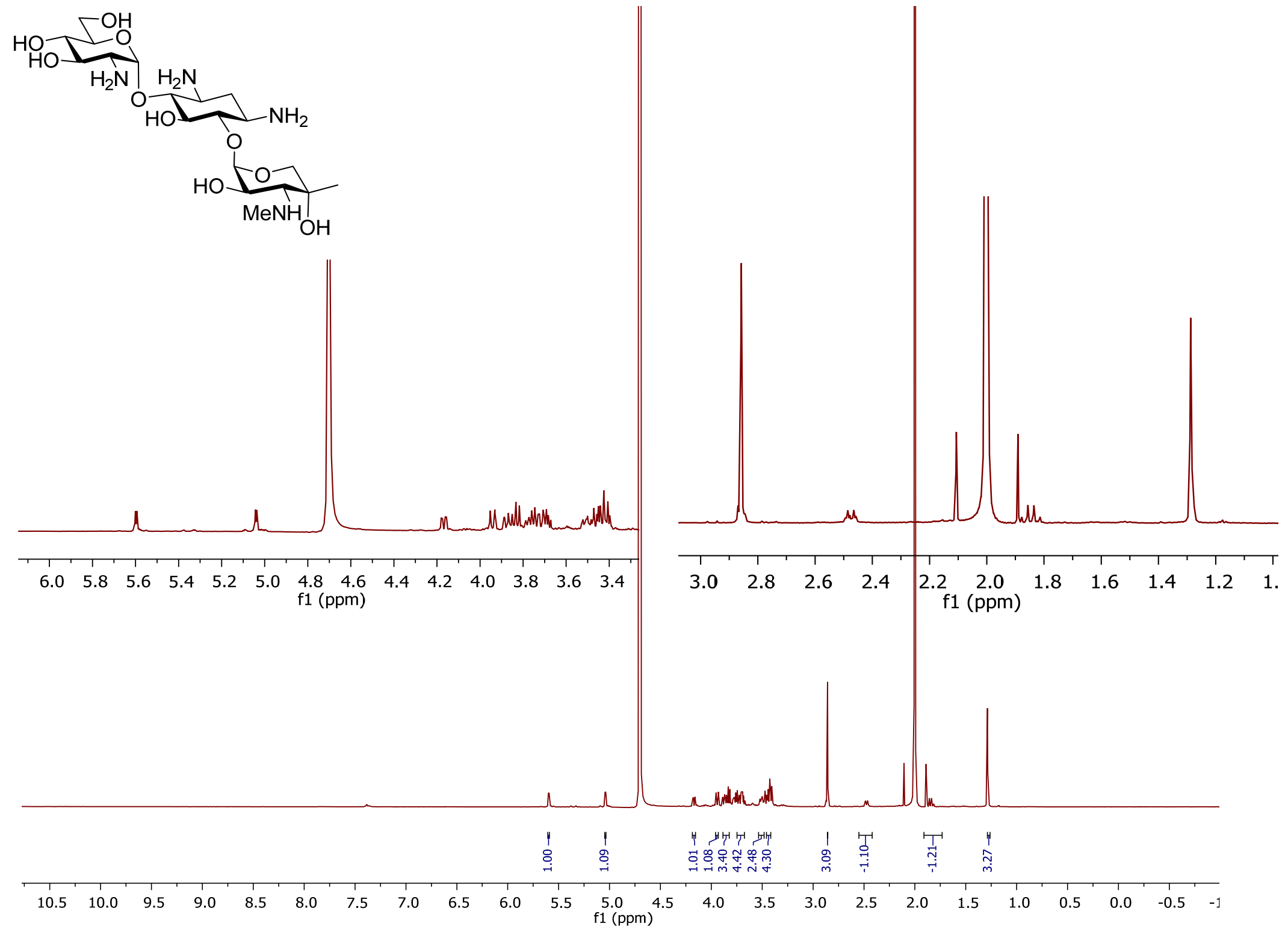

${ }^{1} \mathrm{H}$ NMR $\left(600 \mathrm{MHz}, \mathrm{D}_{2} \mathrm{O}\right)$ of Gentamicin X2 tetraaacetate salt (8). 

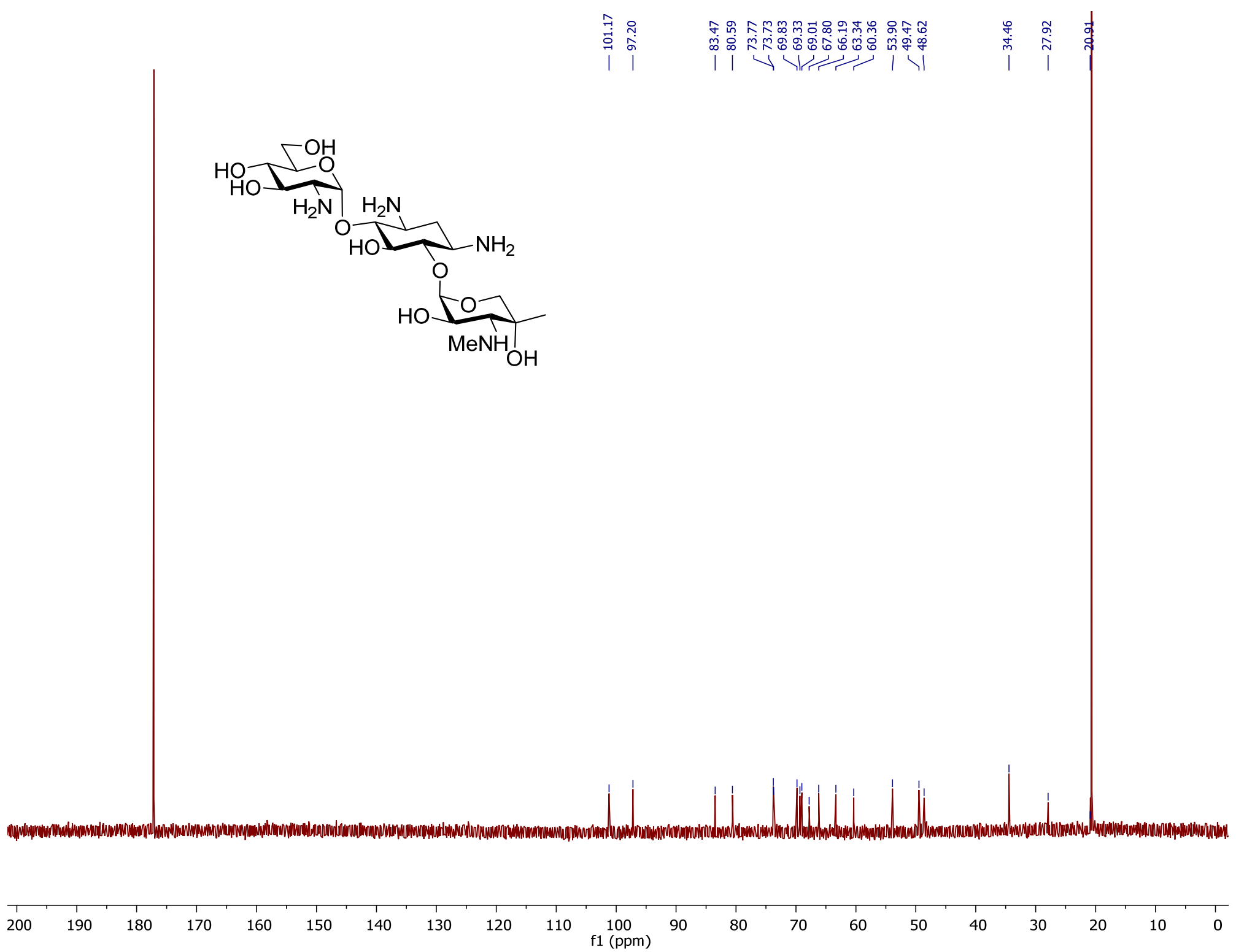

${ }^{13} \mathrm{C}$ NMR (150 MHz, $\left.\mathrm{D}_{2} \mathrm{O}\right)$ of Gentamicin X2 tetraaacetate salt (8). 